Mireles-Velázquez, D. Rosales-Contreras, G.I., ConejerosVargas, C.A., Solís-Marín, F.A., \& Granja-Fernández, R. (2021). Los ofiuroideos (Echinodermata: Ophiuroidea) de la bahía de Chamela, Jalisco, México. Revista de Biología Tropical, 69(S1), 312333. DOI 10.15517/rbt.v69iSuppl.1.46364

\title{
Los ofiuroideos (Echinodermata: Ophiuroidea) de la bahía de Chamela, Jalisco, México
}

\author{
Daniel Mireles-Velázquez ${ }^{1 *}$ \\ Gloria Itzel Rosales-Contreras ${ }^{2}$ \\ Carlos Andrés Conejeros-Vargas ${ }^{2}$ \\ Francisco Alonso Solís-Marín ${ }^{3}$ \\ Rebeca Granja-Fernández ${ }^{4}$
}

1. Licenciatura en Biología, Facultad de Ciencias, Universidad Nacional Autónoma de México, Av. Universidad, Circuito Exterior s/n, Alcaldía Coyoacán, Ciudad de México, México; danielmireles@ciencias.unam.mx (*Correspondencia).

2. Posgrado en Ciencias del Mar y Limnología, Instituto de Ciencias del Mar y Limnología, Universidad Nacional Autónoma de México, Av. Universidad, Circuito Exterior s/n, Alcaldía Coyoacán, Ciudad de México, México; roscongloitz@hotmail.com; conejeros@ciencias.unam.mx

3. Laboratorio de Sistemática y Ecología de Equinodermos, Instituto de Ciencias del Mar y Limnología, Universidad Nacional Autónoma de México, Av. Universidad, Circuito Exterior s/n, Alcaldía Coyoacán, Ciudad de México, México; fasolis@cmarl.unam.mx

4. Laboratorio de Ecología Marina, Centro Universitario de la Costa, Universidad de Guadalajara, Av. Universidad, Puerto Vallarta, Jalisco, México; beckygranja@gmail.com

Recibido 07-VII-2020. Corregido 25-VIII-2020. Aceptado 23-X-2020.

\section{ABSTRACT \\ Ophiuroids (Echinodermata: Ophiuroidea) from Chamela bay, Jalisco, Mexico}

\begin{abstract}
Introduction: Chamela bay is located in the central portion of the coast from the Jalisco state, Mexico and is an important protected natural area. Despite this, the study of echinoderms in the area is incipient. Objective: To provide an updated checklist, descriptions of the species and a taxonomic key of the ophiuroids from Chamela bay. Methods: We collected specimens from 1998 to 2018, and also analyzed information from the literature and specimens deposited at international scientific collections from Chamela bay. Results: 19 species of ophiuroids are reported for the study area, distributed in two orders, seven families, and 11 genera. Seven of these correspond to new records for the area, one to a new record for Jalisco state, and two possibly represent new species. The biodiversity of ophiuroids in Chamela bay represents $15.2 \%$ of ophiuroid species from the Mexican Pacific and $73 \%$ from Jalisco. The number of known echinoderms in Chamela bay is increased to 48 species. Conclusions: The most complete checklist of the class Ophiuroidea in Chamela bay, Jalisco, is presented. The information provided can be used for other areas of the eastern Pacific. Chamela bay is important in terms of species richness due to its environmental heterogeneity and numerous islands.
\end{abstract}

Key words: taxonomic key; Mexican Pacific; biodiversity; new records; morphology; taxonomy.

La bahía de Chamela se encuentra ubicada en la porción central de la costa del estado de Jalisco, entre Punta Rivas (19³4’36” N \& $105^{\circ} 08^{\prime} 33^{\prime \prime}$ W) y Punta Chamela (19³0'52" $\left.\mathrm{N} \& 105^{\circ} 04^{\prime} 56^{\prime \prime} \mathrm{W}\right)$, México (López-Uriarte et al., 2009). Esta bahía se encuentra representada 
por diez islas: Pajarera y Cocinas son las de mayor tamaño, y se encuentran equidistantes con respecto a los dos extremos de la bahía (Ríos-Jara et al., 2013), mientras que el resto de las islas (San Agustín, San Pedro, San Andrés, El Mamut, La Colorada, Los Anegados, El Novillo y La Negrita) son de menor tamaño y se encuentran localizadas a lo largo de la bahía (Fig. 1). Estas islas constituyen el primer santuario en México decretado bajo esta categoría e incorporado al Sistema Nacional de Áreas Naturales Protegidas (Miranda, Ambriz, Valencia, Sánchez \& Szekely, 2011).

En el Pacífico mexicano existen numerosos trabajos taxonómicos de ofiuroideos (e.g. Le Conte, 1851; Verrill, 1867; Clark, 1913; Ziesenhenne, 1940; Caso, 1951) y como resultado, se tienen reportadas 125 especies (Granja-Fernández, Herrero-Pérezrul, López-Pérez, Hernández-Morales, \& Rangel-Solís, 2015); de las anteriores, únicamente se tienen registradas 26 para el estado de Jalisco (Granja-Fernández et al., 2017). En la costa de Jalisco en general los trabajos en equinodermos han sido escasos, particularmente para la bahía de Chamela.

A finales del siglo XX, se realizó el primer trabajo de equinodermos exclusivo para la isla Cocinas en la bahía de Chamela, elaborado por Frontana-Uribe y Solís-Marín (1999), quienes presentaron un listado taxonómico con 22 especies de equinodermos. Catorce años después, se publicó el más completo listado taxonómico de los equinodermos de la bahía de Chamela, en este trabajo Ríos-Jara et al. (2013) describieron la composición taxonómica de los equinodermos de los ambientes intermareales y submareales de 14 localidades dentro de la bahía, incluyendo sus principales islas e islotes; en total, reportaron 40 especies de equinodermos.

El primer trabajo de ofiuroideos para la bahía de Chamela corresponde a Clark (1940) quien reportó a Ophioderma variegatum y Ophiolepis variegata, como resultado de una expedición realizada por el buque de investigación Zaca. Posteriormente, Luke (1982) anexó al inventario dos registros más, Ophiocomella

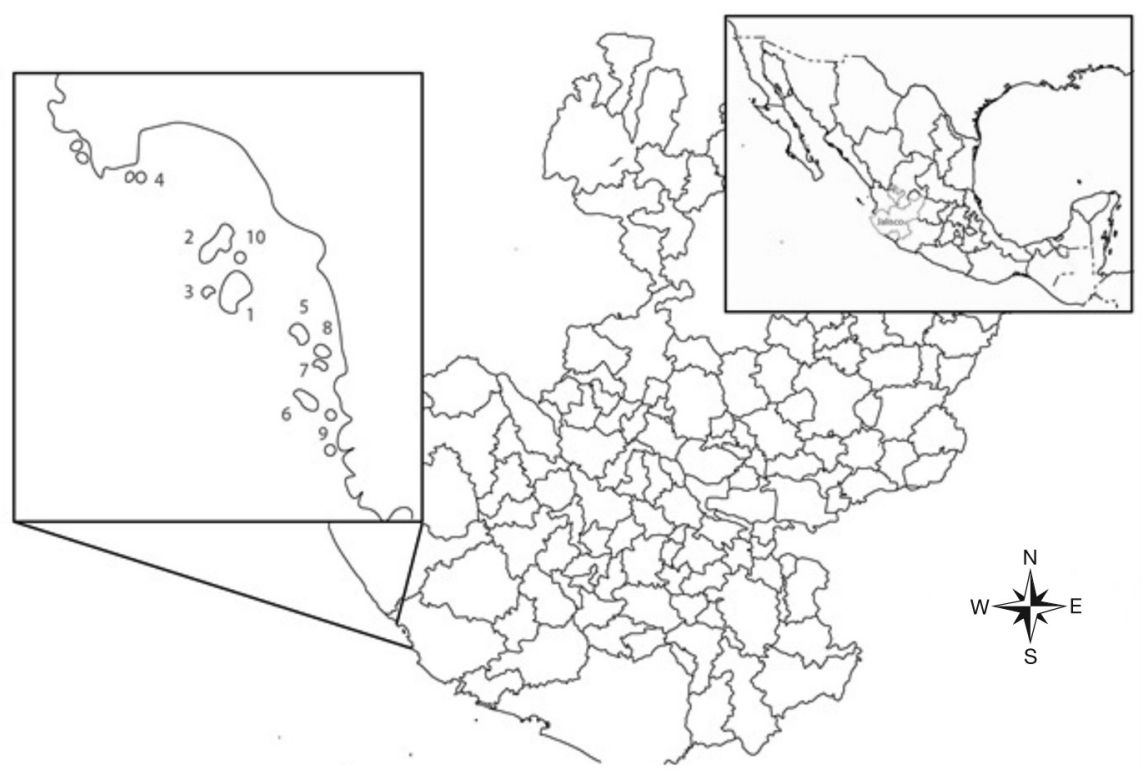

Fig. 1. Mapa de localización de la bahía de Chamela en el Pacífico mexicano y sus islas: 1) isla Cocinas; 2) isla Pajarera; 3) isla El Mamut; 4) islotes Los Anegados; 5) isla La Colorada; 6) isla San Andrés; 7) isla San Pedro; 8) isla San Agustín; 9) isla La Negrita; 10) isla El Novillo.

Fig. 1. Map showing the location of Chamela bay in the Mexican Pacific and its islands: 1) Cocinas Island; 2) Pajarera Island; 3) El Mamut Island; 4) Los Anegados Islets; 5) La Colorada Island; 6) San Andres Island; 7) San Pedro Island; 8) San Agustin Island; 9) La Negrita Island; 10) El Novillo Island. 
alexandri y Ophiothela mirabilis. Dos décadas más tarde, López-Uriarte et al. (2009) llevaron a cabo un estudio de macroinvertebrados bentónicos en la localidad Punta La Rosada, quienes encontraron nuevos registros de ofiuroideos para la bahía (Ophiactis savignyi, Ophiocoma aethiops y Ophioderma panamense). Ríos-Jara et al. (2013) realizaron recolectas en diversas islas en la bahía de Chamela, encontrando 10 especies de ofiuroideos (y una más de identidad no corroborada), de las cuales Ophiactis simplex, Ophionereis annulata, Ophiophragmus marginatus y Ophiothrix (Ophiothrix) spicula$t a$, correspondieron a nuevos registros. Finalmente, Granja-Fernández et al. (2014, 2017) recolectaron siete especies de ofiuroideos en cinco localidades de la bahía. En resumen, hasta la fecha se han reportado 11 especies de ofiuroideos para bahía de Chamela, pero ningún trabajo ha realizado una revisión taxonómica detallada para el grupo en esta área.

Con la finalidad de enriquecer el conocimiento de la diversidad y distribución de la clase Ophiuroidea en la bahía y para el estado de Jalisco, el objetivo de este trabajo es presentar un listado actualizado, descripciones taxonómicas, así como una clave de identificación de las especies de ofiuroideos recolectadas en la bahía de Chamela.

\section{MATERIALES Y MÉTODOS}

Para la elaboración de este trabajo se llevó a cabo una búsqueda bibliográfica de las especies de ofiuroideos reportadas previamente para la bahía de Chamela. Adicionalmente, se llevaron a cabo 13 salidas al campo en los años $1998,2004,2007-2014$ y 2016-2018, donde se recolectaron ejemplares de la clase. El material recolectado se encuentra depositado en la Colección Nacional de Equinodermos "Dra. María Elena Caso Muñoz" del Instituto de Ciencias del Mar y Limnología de la Universidad Nacional Autónoma de México, México (ICML-UNAM). Los muestreos se llevaron a cabo mediante buceo libre y buceo SCUBA, a profundidades que variaron entre 2 y $10 \mathrm{~m}$, en un total de ocho localidades (Tabla 1). De cada muestra se obtuvo información adicional a los ejemplares que incluye tipo de sustrato, hábitat y profundidad.

De manera complementaria, se revisó el material depositado en las colecciones: Museum of Comparative Zoology, Harvard University, Cambridge, Massachusetts, E.U.A. (MCZ), y Smithsonian National Museum of Natural History, Washington, D.C., E.U.A. (USNM). Asimismo, se corroboró la identidad de todas las especies respecto al material tipo

TABLA 1

Localidades de muestreo entre los años 1998 a 2018 por el Laboratorio de Sistemática y Ecología de Equinodermos

TABLE 1

Sampling localities between the years 1998 to 2018 carried out by the Laboratorio de Sistemática y Ecología de Equinodermos

\begin{tabular}{|c|c|c|c|}
\hline Localidad & Coordenadas & Profundidad & Tipo de sustrato \\
\hline Isla Cocinas & $19^{\circ} 32^{\prime} 46.2^{\prime \prime} \mathrm{N} / 105^{\circ} 06^{\prime} 32^{\prime \prime} \mathrm{W}$ & $6 \mathrm{~m}$ & Rocoso / Arenoso / Madera sumergida \\
\hline Playa Huamuchil, isla Cocinas & $19^{\circ} 33^{\prime} 1.99^{\prime \prime} \mathrm{N} / 105^{\circ} 06^{\prime} 26.2^{\prime \prime} \mathrm{W}$ & $3 \mathrm{~m}$ & Rocoso / Arenoso / Madera sumergida \\
\hline Punta Noroeste, isla Cocinas & $19^{\circ} 33^{\prime} 2^{\prime \prime} \mathrm{N} / 105^{\circ} 06^{\prime} 23^{\prime \prime} \mathrm{W}$ & $4 \mathrm{~m}$ & Rocoso \\
\hline Isla Pajarera & $19^{\circ} 33^{\prime} 23.2^{\prime \prime} \mathrm{N} / 105^{\circ} 06^{\prime} 48.7 \mathrm{~W}$ & $8 \mathrm{~m}$ & Rocoso / Algas \\
\hline Isla El Mamut & $19^{\circ} 32^{\prime} 57.5^{\prime \prime} \mathrm{N} / 105^{\circ} 06^{\prime} 50.3^{\prime \prime} \mathrm{W}$ & $10 \mathrm{~m}$ & Rocoso / Algas \\
\hline Isla La Negrita & $19^{\circ} 31^{\prime} 19.1^{\prime \prime} \mathrm{N} / 105^{\circ} 05^{\prime} 6.7^{\prime \prime} \mathrm{W}$ & $2 \mathrm{~m}$ & Rocoso \\
\hline Isla San Pedro & $19^{\circ} 33^{\prime} 13.2^{\prime \prime} \mathrm{N} / 105^{\circ} 06^{\prime} 37.7^{\prime \prime} \mathrm{W}$ & $5 \mathrm{~m}$ & Rocoso \\
\hline Isla La Colorada & $19^{\circ} 32^{\prime} 23.0^{\prime \prime} \mathrm{N} / 105^{\circ} 05^{\prime} 31.8^{\prime \prime} \mathrm{W}$ & $8 \mathrm{~m}$ & Rocoso \\
\hline
\end{tabular}


TABLA 2

Especies de ofiuroideos de la bahía de Chamela, indicándose la fuente de registro, nuevos registros y el número de especímenes examinados

TABLE 2

Ophiuroid species from Chamela bay, indicating the source of record, new records, and the number of examined specimens

\begin{tabular}{|c|c|c|c|c|c|c|}
\hline Especie & $\begin{array}{c}\text { Registro } \\
\text { ICML-UNAM }\end{array}$ & $\begin{array}{l}\text { Registro } \\
\text { MCZ }\end{array}$ & $\begin{array}{l}\text { Registro } \\
\text { USNM }\end{array}$ & $\begin{array}{l}\text { Registro } \\
\text { literatura }\end{array}$ & $\begin{array}{l}\text { Nuevos } \\
\text { registros }\end{array}$ & $\begin{array}{c}\text { No. especímenes } \\
\text { examinados }\end{array}$ \\
\hline Ophiocnida hispida & $\mathrm{X}$ & & & & $\mathrm{X}$ & 5 \\
\hline Ophiophragmus marginatus & & & & $\mathrm{x}$ & & \\
\hline Ophiophragmus sp. & $\mathrm{X}$ & & & & $\mathrm{X}$ & 2 \\
\hline Ophiothrix (Ophiothrix) spiculata & $\mathrm{X}$ & & & $\mathrm{X}$ & & 101 \\
\hline Ophiothrix sp. & $\mathrm{X}$ & & & & $\mathrm{X}$ & 7 \\
\hline Ophiothela mirabilis & $\mathrm{X}$ & & & $\mathrm{x}$ & & 691 \\
\hline Ophiactis savignyi & $\mathrm{X}$ & & & $\mathrm{x}$ & & 832 \\
\hline Ophiactis simplex & $\mathrm{X}$ & & & $\mathrm{X}$ & & 25 \\
\hline Ophionereis annulata & $\mathrm{X}$ & & & $\mathrm{X}$ & & 33 \\
\hline Ophiolepis pacifica & $\mathrm{X}$ & & & & $\mathrm{X}$ & 3 \\
\hline Ophiolepis variegata & & & & $\mathrm{X}$ & & \\
\hline Ophiolepis plateia & $\mathrm{x}$ & & & & $\mathrm{X}$ & 17 \\
\hline Ophiocoma aethiops & $\mathrm{X}$ & & & $\mathrm{x}$ & & 85 \\
\hline Ophiocomella alexandri & $\mathrm{x}$ & $\mathrm{X}$ & & $\mathrm{x}$ & & 176 \\
\hline Ophioderma variegatum & & $\mathrm{X}$ & & $\mathrm{X}$ & & 1 \\
\hline Ophioderma panamense & $\mathrm{x}$ & $\mathrm{x}$ & & $\mathrm{X}$ & & 37 \\
\hline Ophioderma teres var. unicolor & $\mathrm{x}$ & $\mathrm{X}$ & & & $\mathrm{x}$ & 12 \\
\hline Ophioderma hendleri & $\mathrm{x}$ & & & & & 1 \\
\hline Diopederma daniana & & & $\mathrm{x}$ & & $\mathrm{x}$ & 1 \\
\hline TOTAL & & & & & & 2029 \\
\hline
\end{tabular}

depositado en las colecciones anteriores y en el Natural History Museum of Los Angeles County, Los Angeles, California, E.U.A. (LACM), y Natural History Museum of Denmark, University of Copenhagen, Copenhagen, Dinamarca (NHMD).

Se corroboró la identidad taxonómica de los ejemplares examinando los caracteres morfológicos externos con ayuda de un microscopio estereoscópico Olympus SZX7. Los caracteres revisados fueron: la ornamentación dorsal y ventral del disco, escudos radiales, placas dorsales, ventrales y accesorias de los brazos, número y forma de las espinas por cada placa lateral de los brazos, número de escamas tentaculares, forma de los escudos orales, escudos adorales, número y forma de papilas orales y dentales. La identificación de los especímenes se basó en los caracteres taxonómicos observados en el material tipo revisado, así como de las claves de identificación y las descripciones contenidas en los trabajos de Gray (1840), Müller y Troschel $(1840,1842)$, Le Conte (1851), Lütken (1856, 1859), Lyman (1865), Ljungman (1866, 1867), Verrill (1867), Matsumoto (1915), Fell (1960), Granja-Fernández et al. (2014), O'Hara, Stöhr, Hugall, Thuy y Martynov (2017) y Hendler (2018).

Con la finalidad de elaborar láminas de identificación de las especies se tomaron fotografías multifocales con un microscopio estereoscópico Leica Z16 APOA en el Laboratorio de la Biodiversidad II del Instituto de Biología (IB), UNAM. Asimismo, se realizó una clave de identificación taxonómica y se elaboraron descripciones para cada una de las especies. Finalmente, con el material examinado se realizó un listado sistemático, basado en 
la clasificación propuesta por O’Hara, Stöhr, Hugall, Thuy, y Martynov (2018) y Stöhr, O’Hara, y Thuy (2020).

\section{RESULTADOS}

Se revisaron taxonómicamente un total de 2029 ejemplares pertenecientes a dos órdenes, siete familias, 11 géneros y 19 especies.
De estos registros, 11 especies ya habían sido previamente reportadas en la literatura, mientras que siete especies corresponden a nuevos registros (Tabla 2).

A continuación, se presenta la clave taxonómica para la identificación de las especies y sus respectivas descripciones taxonómicas. Acrónimos usados: DD = Diámetro del disco; $\mathrm{LB}=$ Longitud del brazo más largo.

\section{Clave taxonómica para la identificación de ofiuroideos de la bahía de Chamela, Jalisco, México}

1 Disco cubierto por escamas, espinas, espineletas, tocones

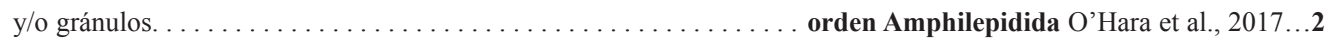

1' Disco cubierto solamente por gránulos . . . . . . . . . . . . . . o orden Ophiacanthida O'Hara et al., 2017...13

2 Papilas infradentales en forma de bloque. . . . . . . . . . . . . . . familia Amphiuridae Ljungman, 1867...3

2' Papilas infradentales ausentes o cuando están presentes, no son en forma de bloque $\ldots \ldots \ldots \ldots \ldots \ldots \ldots \ldots$

3 Disco dorsal con pequeñas escamas imbricadas y espinas (género Ophiocnida Lyman, 1865); escudos radiales pequeños, triangulares. Escudos orales pequeños con forma de diamante, con proyección distal corta. Brazos largos con

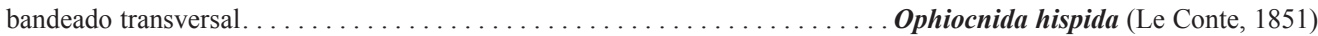

3. Disco con escamas imbricadas y espinas limitadas al margen del disco. . . género Ophiophragmus Lyman, 1865...4

4 Disco con pequeñas escamas imbricadas. Hasta 12 espinas pequeñas por interradio. Escudos radiales solo en contacto en la parte distal, el resto separado por numerosas escamas. Escudos orales con proyección distal $\ldots \ldots \ldots \ldots \ldots \ldots \ldots \ldots \ldots \ldots \ldots \ldots \ldots \ldots \ldots \ldots$ Ophiophragmus $\mathbf{s p .}$

4' Disco con escamas grandes imbricadas. Hasta 25 espinas pequeñas por interradio. Escudos radiales unidos en toda su longitud, con una escama insertada proximalmente. Escudos orales sin

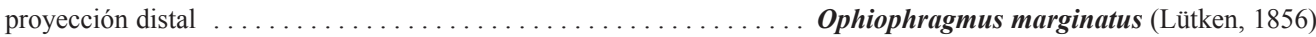

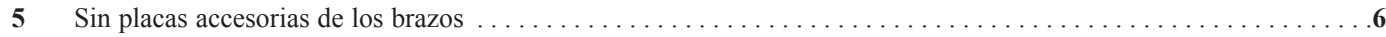

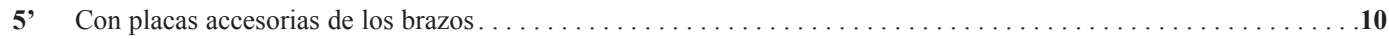

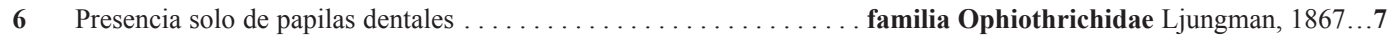

6' Presencia de una escama bucal y una espina del escudo adoral . . . . . . . familia Ophiactidae Matsumoto, $1915 \ldots 9$

7 Cinco brazos. Disco dorsal con espineletas y/o tocones. Brazos dorsal sin ornamentación. Espinas de los brazos generalmente hialinas o vítreas $\ldots \ldots \ldots \ldots \ldots \ldots \ldots \ldots \ldots$ género Ophiothrix Müller \& Troschel, 1840...8

7. Seis brazos (raramente cinco). Disco dorsal con gránulos. Brazos dorsal cubiertos por gránulos. Espinas de los brazos no hialinas o vítreas, a excepción de la más ventral (género Ophiothela Verrill, 1867); brazos enrollados. Mandíbula y placas ventrales de los brazos cubiertas por piel. Coloración rosácea-púrpura . . Ophiothela mirabilis Verrill, 1867

8 Disco cubierto por tocones y espineletas. Espinas de los brazos largas,

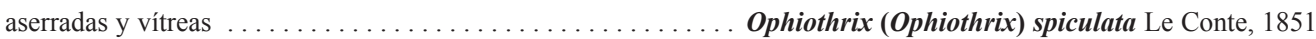

8. Disco cubierto por tocones cortos y robustos. Espinas de los brazos largas, robustas y aserradas

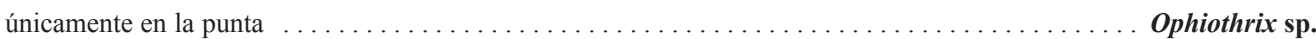

9 Margen del disco cubierto por pequeñas espinas. Escudos radiales largos, equivalentes a $3 / 4$ del diámetro del disco. Presencia de dos papilas orales sensu lato y ocasionalmente una tercera papila oral

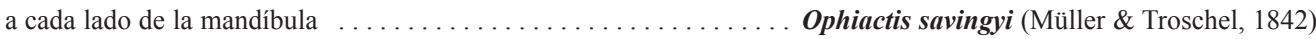

9' Margen del disco ocasionalmente cubierto por pequeñas espinas. Escudos radiales pequeños de $1 / 4$ del diámetro del disco. Una papila oral sensu lato a cada lado de la mandíbula . . . . . . . . . . Ophiactis simplex (Le Conte, 1851) 
10 Disco con escamas muy finas e imbricadas. Escudos radiales pequeños (familia Ophionereididae Ljungman, 1867); escudos radiales cubiertos por escamas. Presencia de osículo de Lyman, espina del escudo adoral, segunda espina del escudo adoral, escama bucal y par de papilas infradentales en el ápice de la mandíbula. Una escama tentacular. Espinas del brazo medianamente largas con respecto a las placas laterales (género Ophionereis Lütken, 1859). Presencia de papilas genitales. Brazos largos y delgados. Coloración amarillo-grisácea, disco beige con reticulación marrón, bandas

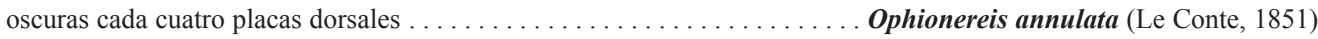

10' Disco con placas grandes. Escudos radiales grandes (familia Ophiolepididae Ljungman, 1867). Presencia de osículo de Lyman, espina del escudo adoral y segunda espina del escudo adoral rectangulares, escama bucal y papila infradental lanceoladas. Dos escamas tentaculares. Espinas del brazo cortas, tres veces más pequeñas

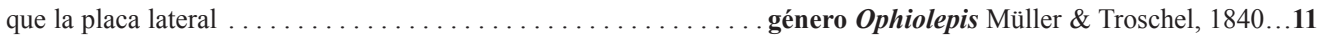

11 Placa central rodeada por cinco escamas triangulares más pequeñas. Escudos radiales medianos ( $1 / 5$ del diámetro del disco), hundidos y triangulares, con muesca en su contorno. Placas accesorias ligeramente visibles en los primeros

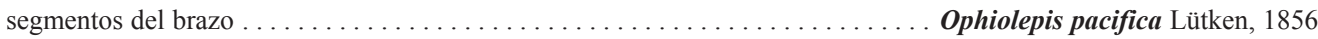

11 Placas primarias muy evidentes. Escudos radiales en forma de gota. Placas accesorias visibles en todos los segmentos

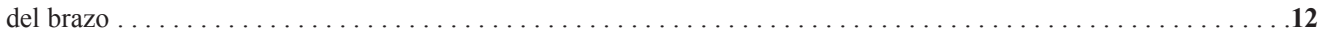

12 Placa central de menor tamaño que las placas primarias. Placa genital abradial dividida en dos segmentos. Coloración

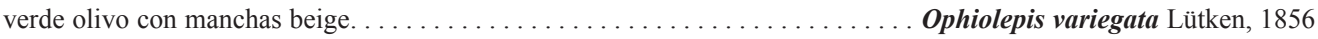

12. Placa central de tamaño similar a las placas primarias. Placa genital abradial dividida en tres segmentos. Coloración

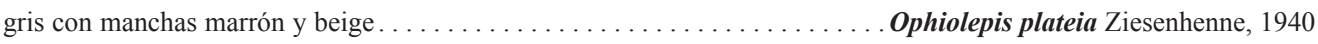

13 Presencia de osículo de Lyman, espina del escudo adoral, segunda espina del escudo adoral, papilas infradentales y cúmulo de papilas dentales. Espinas de los brazos largas y robustas.

Dos hendiduras genitales por interradio . . . . . . . . . . . . Familia Ophiocomidae (Ljungman, 1867)...14

13. Presencia de osículo de Lyman, espina del escudo adoral, segunda espina del escudo adoral, papilas orales laterales, papilas infradentales y papilas dentales (no en cúmulo). Espinas de los brazos cortas con respecto a la placa dorsal. Dos pares de hendiduras genitales pequeñas por interradio con respecto a los segmentos del brazo . . . . . Familia

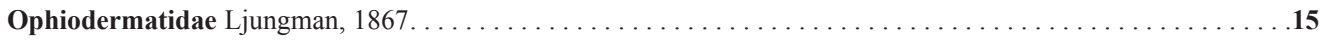

14 Gránulos del disco redondeados. Escudos orales ovalados (género Ophiocoma L. Agassiz, 1836); placas dorsales de los brazos ovaladas e irregulares. Espinas de los brazos en patrón intercalado de tres y cuatro. Coloración negra o

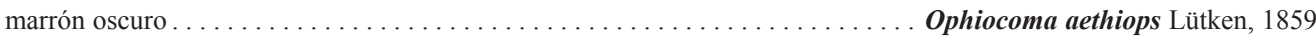

14' Gránulos del disco alargados. Escudos orales redondeados (género Ophiocomella A.H. Clark, 1936); placas dorsales de los brazos en forma de corazón y regulares. Hasta seis espinas en los brazos. Coloración marrón claro, brazos ventral con una línea longitudinal beige $\ldots \ldots \ldots \ldots \ldots \ldots \ldots \ldots \ldots$ Ophiocomella alexandri $($ Lyman, 1860)

15 Disco cubierto por gránulos medianos-grandes; cuatro hendiduras genitales en

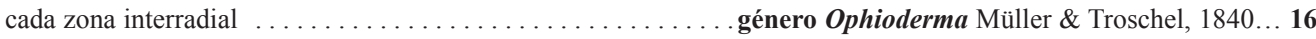

15. Disco cubierto por gránulos pequeños. Cuatro hendiduras genitales, dos en la parte ventral y dos en la parte marginal del disco en vista dorsal (género Diopederma H.L. Clark, 1913); escudos radiales ovalados. Escudos orales ovalados y grandes. Coloración dorsal marrón oscuro con manchas beige . . . . . . . . Diopederma daniana (Verrill, 1867)

16 Escudos radiales desnudos. Escudos adorales cubiertos por gránulos $\ldots \ldots \ldots \ldots \ldots \ldots \ldots \ldots \ldots \ldots$

16' Escudos radiales cubiertos por gránulos. Escudos adorales descubiertos $\ldots \ldots \ldots \ldots \ldots \ldots \ldots \ldots \ldots \ldots$

17 Placas dorsales de los brazos rara vez llegan a estar fragmentadas en dos. De 10 a 11 espinas de los brazos, en algunas ocasiones llegan a presentar 12. Coloración marrón-verdoso,

con manchas dispersas marrón y blanco . . . . . . . . . . . . . . . Ophioderma panamense Lütken, 1859

17. Placas dorsales fragmentadas, llegándose a dividir desde dos hasta cuatro fragmentos. De siete a ocho espinas por cada segmento del brazo. Coloración marrón oscuro

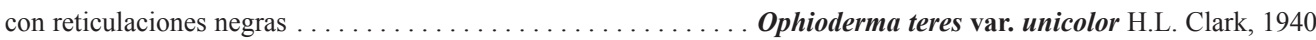

18 Escudos orales ovalados, grandes ( $1 / 7$ del diámetro del disco). De siete a ocho espinas por cada placa lateral de los

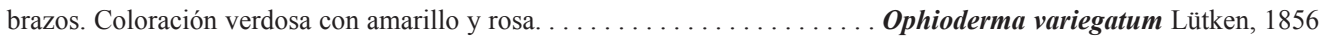

18' Escudos orales triangulares con bordes redondos, pequeños ( $1 / 7$ del diámetro del disco). De ocho a 10 espinas cortas por cada placa lateral de los brazos. Coloración marrón oscuro

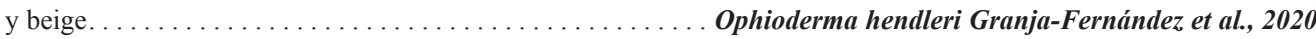


Filo Echinodermata Bruguière, 1791

Clase Ophiuroidea Gray, 1840

Orden Ophiacanthida O'Hara, Hugall, Thuy,

Stöhr \& Martynov, 2017

Familia Ophiodermatidae Ljungman, 1867

Género Ophioderma Müller \& Troschel, 1840 Ophioderma hendleri

Granja-Fernández et al., 2020

(Fig. 2A, 2B)

Descripción (ICML-UNAM 18331): DD $=10 \mathrm{~mm}, \mathrm{LB}=51 \mathrm{~mm}$. Disco pentagonal cubierto por gránulos redondeados. Escudos radiales cubiertos por gránulos. Interradio ventral cubierto por granulación. Escudos orales triangulares con bordes redondeados, más anchos que largos. Escudos adorales pequeños (1/14 del DD), desnudos, triangulares, separados entre sí. Presenta osículo de Lyman, una espina del escudo adoral, una segunda espina del escudo adoral, cuatro papilas orales laterales, una papila infradental a cada lado de la mandíbula y dos papilas dentales en el ápice de la mandíbula. Cinco brazos delgados y alargados. Placas dorsales de los brazos en forma de trapecio con bordes redondos, más anchas (1 $\mathrm{mm})$ que largas $(0.6 \mathrm{~mm})$. Placas ventrales de los brazos cuadrangulares con bordes redondeados, casi igual de anchas que largas. De ocho a 10 espinas de los brazos cortas, la espina más ventral es la más larga llegando a tocar la siguiente escama tentacular, la espina más dorsal es la de menor tamaño. Dos escamas tentaculares, la escama adradial es ligeramente más larga y lanceolada. Coloración in vivo: disco dorsal marrón con pequeñas manchas beige; superficie dorsal de los brazos marrón, cada cuatro segmentos presentan una coloración beige la cual cubre de dos a tres segmentos.

Distribución local: isla Cocinas.

Ophioderma panamense Lütken, 1859

(Fig. 2C, 2D)

Descripción (ICML-UNAM 13280): DD $=23.5 \mathrm{~mm}, \mathrm{LB}=66.0 \mathrm{~mm}$. Disco redondo a rosetado, cubierto por finos gránulos. Escudos radiales ovoides y desnudos. Interradio ventral cubierto por gránulos. Escudos orales triangulares con bordes redondos, más anchos que largos. Escudos adorales cubiertos por gránulos. Presencia de osículo de Lyman, espina del escudo adoral, una segunda espina del escudo adoral, tres papilas laterales orales, una papila infradental en cada lado de la mandíbula y tres papilas dentales en el ápice de la mandíbula. Cinco brazos robustos y alargados. Placas dorsales de los brazos ovaladas, irregulares, más anchas que largas; algunas pueden estar fragmentadas. Placas ventrales de los brazos de forma cuadrangular con ángulos redondeados, ligeramente más anchas que largas. Entre la primera y segunda placa ventral de los brazos se encuentran un par de poros. De diez a 11 espinas de los brazos, en algunas ocasiones llegan a presentar 12. Dos escamas tentaculares, la abradial es dos veces más ancha que la adradial. Coloración in vivo: superficie dorsal del cuerpo de color marrón-verde olivo, generalmente con manchas de color marrón y blanco en el disco.

Distribución local: isla Cocinas, isla Pajarera, isla El Mamut.

\section{Ophioderma teres var. unicolor}

H.L. Clark, 1940

(Fig. 2E, 2F)

Descripción (ICML-UNAM 18365): DD $=22.0 \mathrm{~mm}, \mathrm{LB}=65.0 \mathrm{~mm}$. Disco redondo a pentagonal, cubierto por granulación fina. Escudos radiales descubiertos, pequeños, ovalados y separados. Interradio ventral cubierto por gránulos. Escudos orales más anchos que largos. Escudos adorales cubiertos por gránulos. Presencia de osículo de Lyman, espina del escudo adoral, una segunda espina del escudo adoral, tres papilas laterales orales, una papila infradental a cada lado de la mandíbula y dos papilas dentales en el ápice de la mandíbula. Cinco brazos robustos y alargados. Placas dorsales de los brazos segmentadas en dos o hasta cuatro fragmentos, puede haber algunas placas sin segmentaciones. Placas ventrales de los brazos cuadradas con bordes redondos, ligeramente más largas que anchas. De siete a ocho 
espinas de los brazos, robustas y cortas. Dos escamas tentaculares, la abradial más pequeña. Coloración in vivo: superficie dorsal del cuerpo marrón oscuro, disco con reticulaciones negras.

Distribución local: isla Cocinas, isla El Mamut.

Comentario: Nuevo registro para la bahía de Chamela y para el estado de Jalisco.

Ophioderma variegatum Lütken, 1856 (Fig. 2G, 2H)

Descripción (ICML-UNAM 3.18.36): $\mathrm{DD}=12.4 \mathrm{~mm}, \mathrm{LB}=62.0 \mathrm{~mm}$. Disco redondo, densamente cubierto por gránulos. Escudos radiales e interradio ventral cubiertos por gránulos. Escudos orales muy grandes, ovalados, más largos que anchos. Escudos adorales triangulares, separados entre sí. Presencia de osículo de Lyman, espina del escudo adoral, una segunda espina del escudo adoral, dos papilas laterales orales, una papila infradental a cada lado de la mandíbula y dos papilas dentales en el ápice de la mandíbula. Cinco brazos largos. Placas dorsales de los brazos trapezoidales, más anchas que largas. Placas ventrales de los brazos cuadradas, ligeramente más anchas que largas. Siete a ocho espinas de los brazos, puntiagudas y muy largas, la más dorsal y la más ventral son las más cortas. Dos escamas tentaculares, la adradial más grande. Coloración in vivo: cuerpo dorsal con superficie dorsal verdosa, con amarillo y rosa; brazos con bandas transversales marrón oscuro.

Distribución local: isla La Negrita.

Género Diopederma H.L. Clark, 1913

Diopederma daniana (Verrill, 1867)

(Fig. 3A, 3B)

Descripción (ICML-UNAM 3.25.2): DD $=14.0 \mathrm{~mm}, \mathrm{LB}=42.0 \mathrm{~mm}$. Disco ligeramente en forma de roseta, densamente cubierto por gránulos. Escudos radiales grandes (1/14 del DD), ovalados, separados. Interradio ventral cubierto por gránulos. Dos hendiduras genitales proyectadas dorsalmente y por debajo de los escudos radiales, rodeadas de gránulos espinosos, y dos hendiduras colocadas en la parte ventral. Escudos orales grandes (1/9 del DD), ovalados, más largos que anchos. Escudos adorales triangulares, más largos que anchos, separados entre sí. Presencia de osículo de Lyman, una espina del escudo adoral, una segunda espina del escudo adoral, dos papilas orales laterales, una papila infradental a cada lado de la mandíbula y dos a tres papilas dentales en el ápice de la mandíbula. Cinco brazos largos, robustos en la base, la cual posee gránulos. Placas dorsales de los brazos trapezoidales, más anchas que largas. Placas ventrales de los brazos octagonales, ligeramente más anchas que largas. Hasta diez espinas puntiagudas y cortas de los brazos, la más dorsal es la más corta. Dos escamas tentaculares, la adradial es más grande. Coloración in vivo: superficie dorsal marrón oscuro, superficie ventral beige-blanquecino; superficie dorsal de los brazos marrón oscuro con manchas amarillas-blanquecinas.

Distribución local: Entre isla Cocinas e isla La Negrita.

Comentario: Nuevo registro para la bahía de Chamela.

Familia Ophiocomidae Ljungman, 1867

Género Ophiocoma L. Agassiz, 1836 Ophiocoma aethiops Lütken, 1859

(Fig. 3C, 3D)

Descripción (ICML-UNAM 10880): DD $=30.9 \mathrm{~mm}, \mathrm{LB}=153.0 \mathrm{~mm}$. Disco redondo cubierto de granulación densa y fina, la cual se extiende hasta la base de los brazos. Escudos radiales e interradio cubiertos por granulación. Escudos orales ovalados, más largos que anchos. Escudos adorales triangulares, dispuestos a un costado de los escudos orales. Presenta osículo de Lyman, espina del escudo adoral, una segunda espina del escudo adoral, una papila infradental a cada lado de la mandíbula y de cinco a nueve papilas dentales. Brazos robustos, largos. Placas dorsales de los brazos ovaladas, irregulares, más anchas que largas; algunas de ellas superpuestas. Placas ventrales de los brazos cuadrangulares, casi igual de anchas que de largas. De tres a cuatro espinas de los brazos 


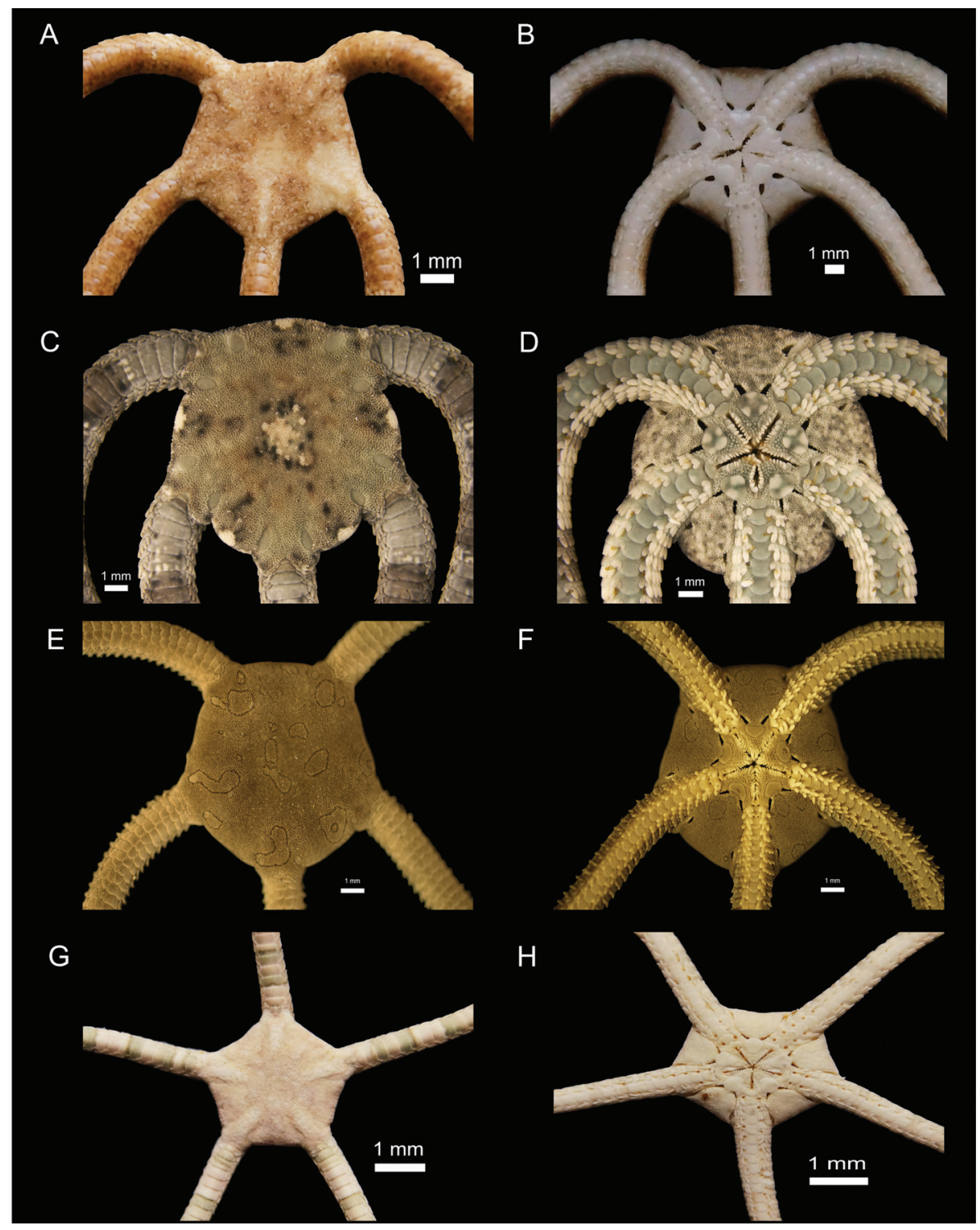

Fig. 2. Ophioderma hendleri (ICML-UNAM 18331). A. Vista dorsal. B. Vista ventral. Ophioderma panamense (ICMLUNAM 13280). C. Vista dorsal. D. Vista ventral. Ophioderma teres var. unicolor (ICML-UNAM 18365). E. Vista dorsal. F. Vista ventral. Ophioderma variegatum (ICML-UNAM 3.18.36). G. Vista dorsal. H. Vista ventral.

Fig. 2. Ophioderma hendleri (ICML-UNAM 18331). A. Dorsal view. B. Ventral view. Ophioderma panamense (ICMLUNAM 13280). C. Dorsal view. D. Ventral view. Ophioderma teres var. unicolor (ICML-UNAM 18365). E. Dorsal view. F. Ventral view. Ophioderma variegatum (ICML-UNAM 3.18.36). G. Dorsal view. H. Ventral view.

alternadas, robustas, de formas variables, la mayoría redondeadas ligeramente aplastadas, algunas curvadas de punta roma. Primeros 15 segmentos del brazo con dos escamas tentaculares regulares ovaladas ligeramente afiladas, posteriormente solo una escama tentacular.
Coloración in vivo: superficie dorsal del cuerpo de color negro o marrón oscuro.

Distribución local: isla Cocinas, isla Pajarera, isla El Mamut, islotes Los Anegados, isla La Colorada, isla San Andrés, isla San Pedro, isla San Agustín. 


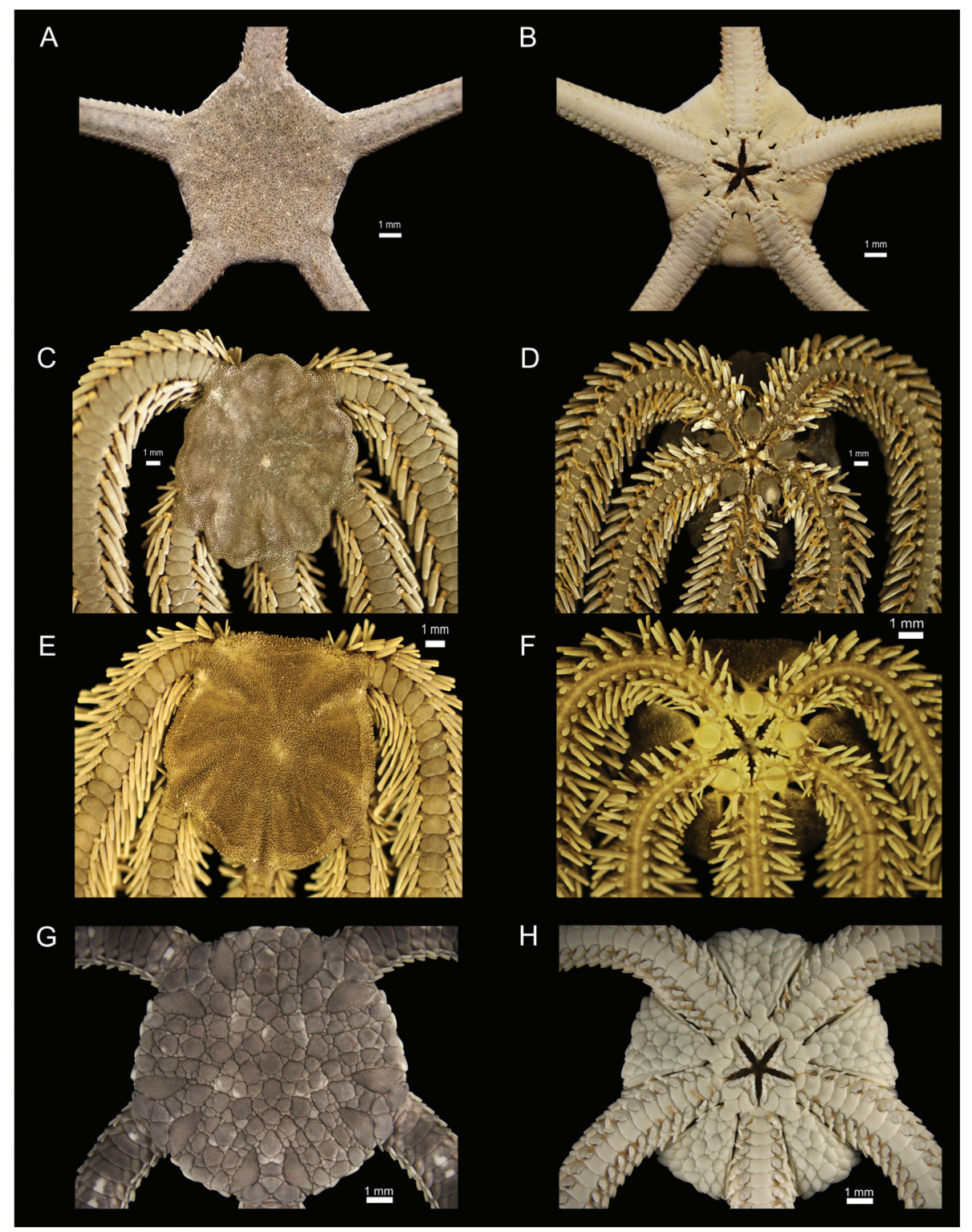

Fig. 3. Diopederma daniana (ICML-UNAM 3.25.2). A. Vista dorsal. B. Vista ventral. Ophiocoma aethiops (ICML-UNAM 10880). C. Vista dorsal. D. Vista ventral. Ophiocomella alexandri (ICML-UNAM 10313). E. Vista dorsal. F. Vista ventral. Ophiolepis pacifica (ICML-UNAM 11948). G. Vista dorsal. H. Vista ventral.

Fig. 3. Diopederma daniana (ICML-UNAM 3.25.2). A. Dorsal view. B. Ventral view. Ophiocoma aethiops (ICML-UNAM 10880). C. Dorsal view. D. Ventral view. Ophiocomella alexandri (ICML-UNAM 10313). E. Dorsal view. F. Ventral view. Ophiolepis pacifica (ICML-UNAM 11948). G. Dorsal view. H. Ventral view. 
Género Ophiocomella A.H. Clark, 1939

Ophiocomella alexandri (Lyman, 1860)

(Fig. 3E, 3F)

Descripción (ICML-UNAM 10313): DD $=28.7 \mathrm{~mm}, \mathrm{LB}=150.0 \mathrm{~mm}$. Disco redondo cubierto por gránulos elongados. Escudos radiales e interradio cubiertos por granulación similar a la parte dorsal. Escudos orales casi circulares. Escudos adorales triangulares a un costado de los escudos orales. Presenta osículo de Lyman, espina del escudo adoral, una segunda espina del escudo adoral y una papila infradental a cada lado de la mandíbula. La mayoría de las papilas dentales se encuentran dispuestas en dos líneas verticales, aproximadamente nueve en total, las de la mitad superior son más grandes que las de la mitad inferior. Brazos robustos y alargados. Placas dorsales de los brazos ovaladas o en forma de corazón. Placas ventrales de los brazos cuadrangulares con bordes redondos, casi igual de anchas que largas. De cuatro a seis espinas de los brazos. Primeros 20 segmentos del brazo con dos escamas tentaculares ovaladas y alargadas, posteriormente solo una escama tentacular. Coloración in vivo: disco color marrón oscuro a claro, placas ventrales con una línea longitudinal beige que corre a lo largo del brazo.

Distribución local: isla Cocinas, isla Pajarera, isla El Mamut, islotes Los Anegados, isla La Colorada, isla San Andrés, isla San Pedro, isla San Agustín, isla El Novillo.

Orden Amphilepidida O'Hara, Hugall, Thuy, Stöhr \& Martynov, 2017

Familia Ophiolepididae Ljungman, 1867

Género Ophiolepis Müller \& Troschel, 1840 Ophiolepis pacifica Lütken, 1856

(Fig. 3G, 3H)

Descripción (ICML-UNAM 11948): DD $=11.4 \mathrm{~mm}, \mathrm{LB}=38.7 \mathrm{~mm}$. Disco redondo, cubierto por placas de forma irregular, grandes y entre estas, placas más pequeñas. Placa primaria evidente, rodeada por cinco placas de menor tamaño de forma triangular. Escudos radiales hundidos y triangulares, más largos que anchos, presentan algunas ornamentaciones en su margen, completamente separados por 18 a 20 placas. Interradio cubierto por placas de tamaño y forma irregular, de menor tamaño que las dorsales. Escudos orales más largos que anchos, de forma pentagonal con alargamiento distal. Escudos adorales triangulares, grandes y en contacto. Presencia de osículo de Lyman, espina del escudo adoral, una segunda espina del escudo adoral, una escama bucal y una papila infradental a cada lado de la mandíbula. Atrás y en medio de las papilas infradentales se encuentra el primer diente ventral. Brazos delgados y relativamente largos. Placas dorsales de los brazos trapezoidales, más anchas que largas. Presencia de una placa accesoria muy pequeña y poco conspicua en los primeros dos o tres segmentos del brazo. Placas ventrales de los brazos semi-pentagonales, más anchas que largas. Cuatro o cinco espinas cilíndricas, muy pequeñas y de tamaño similar. Dos escamas tentaculares ovaladas. Coloración in vivo: superficie dorsal marrón oscuro, superficie ventral beige-blanquecino.

Distribución local: isla Cocinas.

Comentario: Nuevo registro para la bahía de Chamela.

Ophiolepis plateia Ziesenhenne, 1940

(Fig. 4A, 4B)

Descripción (ICML-UNAM 11971): DD $=10.8 \mathrm{~mm}, \mathrm{LB}=31.3 \mathrm{~mm}$. Disco redondo cubierto por placas muy grandes $(1 / 6$ del DD) rodeadas por placas más pequeñas ( $1 / 28 \mathrm{del} \mathrm{DD})$. Placas primarias grandes y en contacto, todas de tamaño similar. Dos placas sobresalientes redondeadas en el margen interradial y por debajo de los escudos radiales. Escudos radiales grandes ( $1 / 5$ del DD), más largos que anchos, separados entre sí por dos placas grandes. Interradio cubierto por placas mucho más pequeñas que las de la parte dorsal. Placa genital abradial dividida en tres segmentos. Escudos orales pentagonales con proyección distal, más largos que anchos. Escudos adorales rectangulares, en contacto. Presencia de osículo de Lyman, espina del escudo adoral, una segunda espina 
del escudo adoral, una escama bucal y una papila infradental a cada lado de la mandíbula. Atrás y en medio de las papilas infradentales se encuentra el primer diente ventral. Placas dorsales del brazo rectangulares, más anchas que largas basalmente, volviéndose triangulares distalmente. Placas ventrales del brazo semipentagonales con bordes redondeados. Dos placas accesorias triangulares y alargadas. Cuatro espinas del brazo cortas, romas, de similar tamaño. Dos escamas tentaculares triangulares casi del mismo tamaño. Coloración in vivo: superficie dorsal grisácea con manchas dispersas de color negro y marrón, superficie ventral beige-blanquecino.

Distribución local: isla Pajarera.

Comentario: Nuevo registro para la bahía de Chamela.

Ophiolepis variegata Lütken, 1856

(Fig. 4C, 4D)

Descripción (ZMUC OPH-454) (modificada de Granja-Fernández, 2019): $\mathrm{DD}=9.0$ $\mathrm{mm}, \mathrm{LB}=22.0 \mathrm{~mm}$. Disco pentagonal cubierto por 15 placas grandes $(1 / 7$ del DD), rodeadas por placas más pequeñas $(1 / 47$ del DD). Placas primarias conspicuas y juntas, placa central de menor tamaño. Escudos radiales grandes ( $1 / 5$ del DD), en forma de gota, más largos que anchos, separados por tres placas. Interradio cubierto por algunas placas grandes. Placa genital abradial dividida en dos segmentos. Escudos orales pentagonales con borde distal alargado, más largos que anchos. Escudos adorales muy cortos, en contacto entre sí. Presencia de osículo de Lyman, espina del escudo adoral, una segunda espina del escudo adoral, una escama bucal y una papila infradental a cada lado de la mandíbula. Atrás y en medio de las papilas infradentales se encuentra el primer diente ventral. Cinco brazos robustos y largos. Placas dorsales de los brazos hexagonales, un poco más largas que anchas. Dos placas accesorias de los brazos alargadas. Placas ventrales de los brazos heptagonales, más anchas que largas. Tres a cuatro espinas de los brazos cortas, puntiagudas, la de en medio de mayor tamaño. Dos escamas tentaculares subtriangulares, la adradial ligeramente más grande. Coloración in vivo: superficie dorsal beige con manchas verde olivo y marrón. Superficie ventral beige-blanquecina. Brazos verde olivo con manchas beige.

Distribución local: Entre isla Pajarera e islotes Los Anegados.

Familia Ophionereididae Ljungman, 1867

Género Ophionereis Lütken, 1859

Ophionereis annulata (Le Conte, 1851)

(Fig. 4E, 4F)

Descripción (ICML-UNAM 12898): DD $=13.5 \mathrm{~mm}, \mathrm{LB}=119.0 \mathrm{~mm}$. Disco redondo cubierto por pequeñas escamas imbricadas ( $1 / 63$ del DD). Escudos radiales muy cortos, separados por escamas y poco visibles. Interradio ventral cubierto por numerosas escamas diminutas. Presencia de papilas genitales redondas. Escudos orales más largos que anchos, redondos en los ángulos laterales. Escudos adorales pequeños, completamente separados entre ellos y dispuestos a los lados del escudo oral. Presencia de osículo de Lyman, espina del escudo adoral, segunda espina del escudo adoral, escama bucal y un par de papilas infradentales en el ápice de la mandíbula. Brazos largos y delgados. Placas dorsales de los brazos hexagonales, ligeramente más anchas que largas. Placas accesorias de los brazos conspicuas en todos los segmentos. Placas ventrales de los brazos cuadrangulares, ligeramente más anchas que largas. Tres espinas largas, con punta roma y de tamaño similar en cada placa lateral del brazo. Una escama tentacular grande y redonda. Coloración in vivo: superficie dorsal beige con una reticulación de color marrón claro, brazos de color beige, cada tres o cuatro placas dorsales presenta una mancha color verde olivo.

Distribución local: isla Cocinas, isla Pajarera, isla El Mamut, islotes Los Anegados, isla La Colorada, isla San Pedro, isla San Agustín, isla La Negrita. 


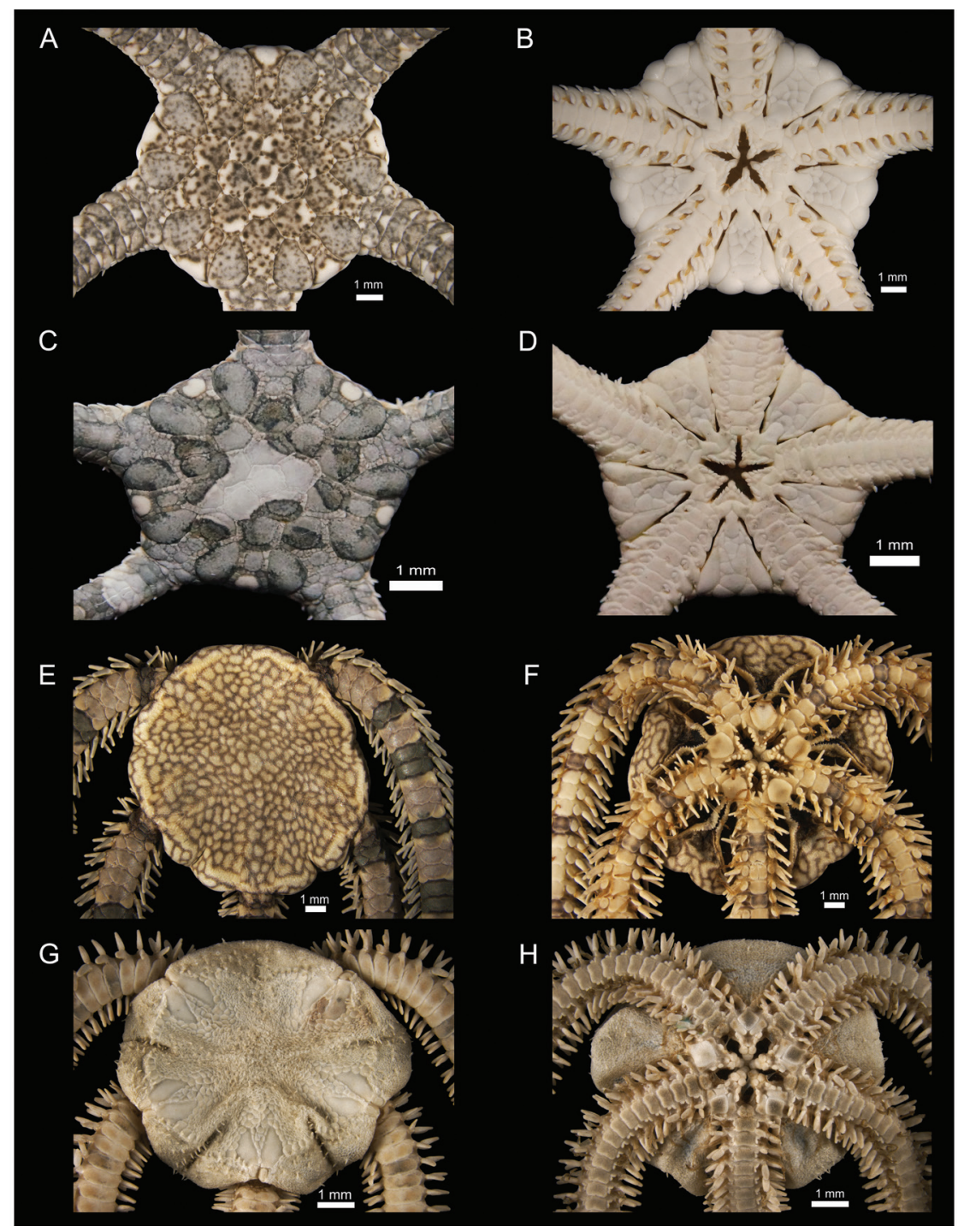

Fig. 4. Ophiolepis plateia (ICML-UNAM 11971). A. Vista dorsal. B. Vista ventral. Ophiolepis variegata (ICML-UNAM 3.26.0). C. Vista dorsal. D. Vista ventral. Ophionereis annulata (ICML-UNAM 12898). E. Vista dorsal. F. Vista ventral. Ophiocnida hispida (ICML-UNAM 18345). G. Vista dorsal. H. Vista ventral.

Fig. 4. Ophiolepis plateia (ICML-UNAM 11971). A. Dorsal view. B. Ventral view. Ophiolepis variegata (ICML-UNAM 3.26.0). C. Dorsal view. D. Ventral view. Ophionereis annulata (ICML-UNAM 12898). E. Dorsal view. F. Ventral view. Ophiocnida hispida (ICML-UNAM 18345). G. Dorsal view. H. Ventral view. 
Familia Amphiuridae Ljungman, 1867

Género Ophiocnida Lyman, 1865

Ophiocnida hispida (Le Conte, 1851)

(Fig. 4G, 4H)

Descripción (ICML-UNAM 18345): DD $=6.9 \mathrm{~mm}, \mathrm{LB}=92.3 \mathrm{~mm}$. Disco redondeado cubierto por escamas pequeñas e imbricadas ( $1 / 70$ del DD), más grandes cerca de los escudos radiales; y espinas cortas, delgadas y afiladas distribuidas dispersamente sobre el disco. Escudos radiales pequeños (1/7 del DD), más largos que anchos, triangulares, separados por escamas. Interradio ventral cubierto por escamas pequeñas e imbricadas. Escudos orales pequeños, con forma de diamante, con ángulos redondeados y proyección distal corta. Escudos adorales pequeños, triangulares, no se encuentran en contacto. Presenta una espina del escudo adoral, una segunda espina del escudo adoral y dos papilas infradentales robustas en el ápice de la mandíbula. Brazos alargados. Placas dorsales de los brazos ovaladas, más anchas que largas. Placas ventrales de los brazos semi-pentagonales, más anchas que largas. Tres espinas de los brazos, cortas, romas de longitudes casi similares. Dos escamas tentaculares anchas y cortas, perpendiculares entre sí. Coloración in vivo: superficie dorsal y ventral beige-marrón claro, brazos con bandas transversales.

Distribución local: isla Cocinas.

Comentario: Nuevo registro para la bahía de Chamela.

Género Ophiophragmus Lyman, 1865

Ophiophragmus marginatus (Lütken, 1856)

Descripción (ZMUC OPH-451) (modificada de Granja-Fernández, 2019): DD = $6.9 \mathrm{~mm}$. Disco redondo, cubierto por escamas grandes ( $1 / 10$ del DD), redondas, de tamaño irregular. Placa central conspicua. Margen del disco con 25 espinas por interradio, ligeramente separadas entre ellas. Escudos radiales cortos, en contacto en toda su longitud, excepto por una escama insertada proximalmente. Interradio ventral cubierto por escamas pequeñas
(1/25 del DD). Escudos orales en forma de diamante, ligeramente más largos que anchos. Escudos adorales triangulares, en contacto entre sí. Presenta una espina del escudo adoral, una segunda espina del escudo adoral y dos papilas infradentales cuadradas en el ápice de la mandíbula. Cinco brazos largos y delgados. Placas dorsales de los brazos ovaladas, más anchas que largas. Placas ventrales de los brazos cuadradas, un poco más anchas que largas. Tres espinas de los brazos cortas, puntiagudas, de tamaño similar. Dos escamas tentaculares perpendiculares. Coloración in vivo: superficie dorsal y ventral beige-marrón claro.

Distribución local: isla Cocinas.

\section{Ophiophragmus sp.}

(Fig. 5A, 5B)

Descripción (ICML-UNAM 18347): DD $=7.0 \mathrm{~mm}, \mathrm{LB}=72.0 \mathrm{~mm}$. Disco redondeado cubierto por pequeñas escamas imbricadas ( $1 / 70$ del DD), las que rodean los escudos radiales de mayor tamaño ( $1 \frac{128}{2}$ del DD). Presenta hasta 12 espinas cortas, delgadas y puntiagudas, sobre el margen del disco. Escudos radiales más largos que anchos, triangulares con bordes redondeados, separados por numerosas escamas, que disminuyen en número hasta solo ser una escama en la parte distal. Interradio ventral cubierto por escamas muy pequeñas e imbricadas $(1 / 140$ del DD). Escudos orales pequeños ( $1 / 9$ del DD), en forma de diamante con una pequeña protuberancia distal. Escudos adorales triangulares con bordes redondeados, ligeramente separados. Presenta una espina del escudo adoral, una segunda espina del escudo adoral y dos papilas infradentales robustas y redondas en el ápice de la mandíbula. Brazos deprimidos y alargados. Placas dorsales de los brazos ovaladas, más anchas que largas. Placas ventrales de los brazos semi-pentagonales, más anchas que largas. Tres espinas de los brazos aplanadas, con punta redondeada. Dos escamas tentaculares cortas, perpendiculares entre sí. Coloración in vivo: disco y brazos de color beige-marrón claro.

Distribución local: isla Cocinas. 


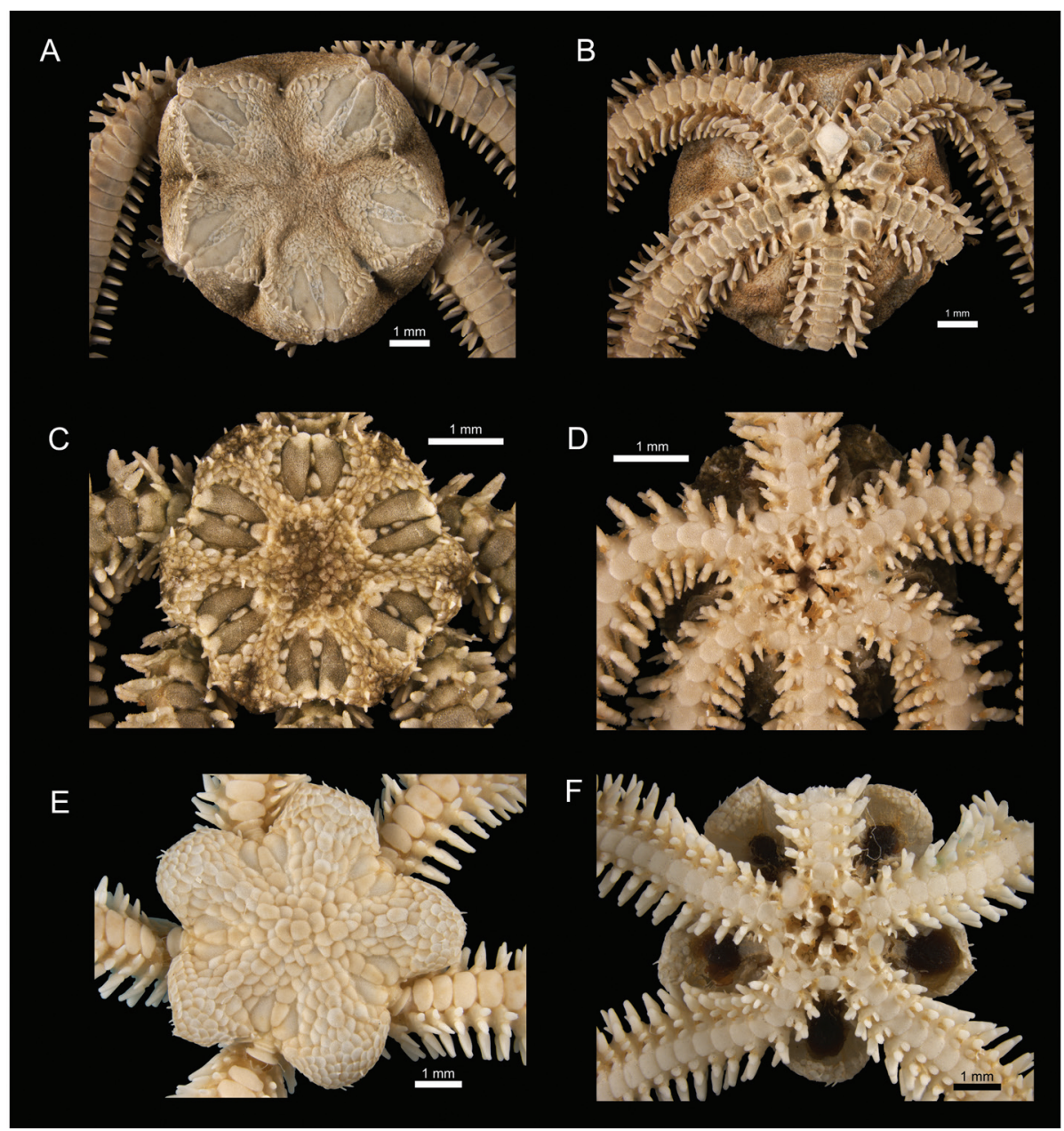

Fig. 5. Ophiophragmus sp. (ICML-UNAM 18347). A. Vista dorsal. B. Vista ventral. Ophiactis savignyi (ICML-UNAM 10319). C. Vista dorsal. D. Vista ventral. Ophiactis simplex (ICML-UNAM 10590). E. Vista dorsal. F. Vista ventral. Fig. 5. Ophiophragmus sp. (ICML-UNAM 18347). A. Dorsal view. B. Ventral view. Ophiactis savignyi (ICML-UNAM 10319). C. Dorsal view. D. Ventral view. Ophiactis simplex (ICML-UNAM 10590). E. Dorsal view. F. Ventral view.

Comentario: Nuevo registro para la bahía de Chamela.

Familia Ophiactidae Matsumoto, 1915

Género Ophiactis Lütken, 1856

Ophiactis savignyi (Müller \& Troschel, 1842) (Fig. 5C, 5D)

Descripción (ICML-UNAM 10319): DD $=3.9 \mathrm{~mm}, \mathrm{LB}=18.4 \mathrm{~mm}$. Disco ligeramente en forma de roseta, cubierto por escamas pequeñas e imbricadas y algunas espinas presentes también en el margen. Escudos radiales muy grandes ( $1 \frac{1}{4}$ del DD), separados por una fila de escamas, solo en contacto en la parte distal. Interradio cubierto por piel y escamas. Escudos orales pequeños, más largos que anchos, rómbicos con bordes redondeados. Escudos adorales triangulares y en contacto. Presencia de dos papilas orales sensu lato y ocasionalmente una tercera papila oral a cada lado de la mandíbula. Seis brazos un poco alargados. Placas dorsales de los brazos ovaladas, más anchas que largas. Placas ventrales de los brazos cuadradas con bordes redondeados, ligeramente más largas que anchas. Siete espinas cortas, gruesas y 
romas en cada placa lateral de los brazos. Una escama tentacular grande y redonda. Coloración in vivo: disco dorsal color verde olivo, con manchas marrón claro, superficie ventral beige, brazos con coloración verde olivo.

Distribución local: isla Cocinas, isla Pajarera, isla La Colorada, isla San Andrés, isla San Pedro, isla San Agustín.

Ophiactis simplex (Le Conte, 1851) (Fig. 5E, 5F)

Descripción (ICML-UNAM 10590): DD $=4.8 \mathrm{~mm}, \mathrm{LB}=24.1 \mathrm{~mm}$. Disco en forma de roseta con escamas grandes, redondas e imbricadas. Margen del disco con cuatro espinas por interradio dorsal. Escudos radiales pequeños y separados por dos escamas grandes en la parte proximal, en contacto solo en la parte más distal. Interradio cubierto por piel y escamas de menor tamaño que las de la parte dorsal. Escudos orales más anchos que largos, ovalados. Escudos adorales alargados, separados entre sí. Presenta una papila oral sensu lato a cada lado de la mandíbula. Cinco o seis brazos un poco alargados. Placas dorsales de los brazos en forma rectangular, con bordes redondos, más anchas que largas. Placas ventrales de los brazos cuadradas con bordes redondos, ligeramente más anchas que largas. Cinco espinas cortas y romas en las placas laterales de los brazos. Una escama tentacular grande y redonda. Coloración in vivo: superficie dorsal y ventral color beige.

Distribución local: isla Cocinas, isla Pajarera, isla La Colorada, isla San Andrés, isla San Pedro, isla San Agustín, isla La Negrita.

Familia Ophiotrichidae Ljungman, 1867

Género Ophiothrix Müller \& Troschel, 1840 Ophiothrix sp.

(Fig. 6A, 6B)

Descripción (ICML-UNAM 18360): DD $=8.1 \mathrm{~mm}, \mathrm{LB}=46.7 \mathrm{~mm}$. Disco en forma de roseta, cubierto por espinas bífidas cortas y robustas. Escudos radiales grandes ( $1 / 3 \mathrm{del} \mathrm{DD})$, más largos que anchos, triangulares con bordes redondeados, separados por una hilera de tocones, en la parte distal en contacto. Interradio cubierto por piel. Brazos un poco alargados y robustos. Escudos orales más anchos que largos, romboidales, con una ligera proyección distal. Escudos adorales triangulares con bordes redondeados, en contacto. Presencia de papilas dentales en forma de racimo. Placas dorsales de los brazos romboidales, con una pequeña proyección distal, ligeramente más anchas que largas. Placas ventrales de los brazos heptagonales, ligeramente más anchas que largas. De siete a ocho espinas largas en cada placa lateral de los brazos, hialinas, más robustas en la base y se adelgazan hacia la punta, lisas, pero con una ligera forma aserrada en la punta, la cual se puede distinguir de manera microscópica; espina más ventral en forma de gancho. Una escama tentacular pequeña y circular. Coloración in vivo: variable con tonos azul cobrizo o violeta.

Distribución local: isla Cocinas.

Comentario: Nuevo registro para la bahía de Chamela.

\section{Ophiothrix (Ophiothrix) spiculata \\ Le Conte, 1851 \\ (Fig. 6C, 6D)}

Descripción (ICML-UNAM 11947): DD $=13.4 \mathrm{~mm}, \mathrm{LB}=57.2 \mathrm{~mm}$. Disco ligeramente rosetado con espineletas aserradas. Escudos radiales conspicuos, más largos que anchos, separados y rodeados por espineletas, en la parte distal en contacto. Interradio cubierto por escamas y espinas cortas. Escudos orales más anchos que largos, con ángulos laterales redondeados. Escudos adorales pequeños, triangulares y en contacto. Presencia de papilas dentales en forma de racimo. Placas dorsales de los brazos romboidales, más anchas que largas. Placas ventrales hexagonales. Siete espinas de los brazos cristalinas y aserradas en cada placa lateral, las dos más ventrales son las más cortas; las subsecuentes aproximadamente el doble de largo que el diámetro del brazo. Una escama tentacular pequeña y circular. Coloración in 


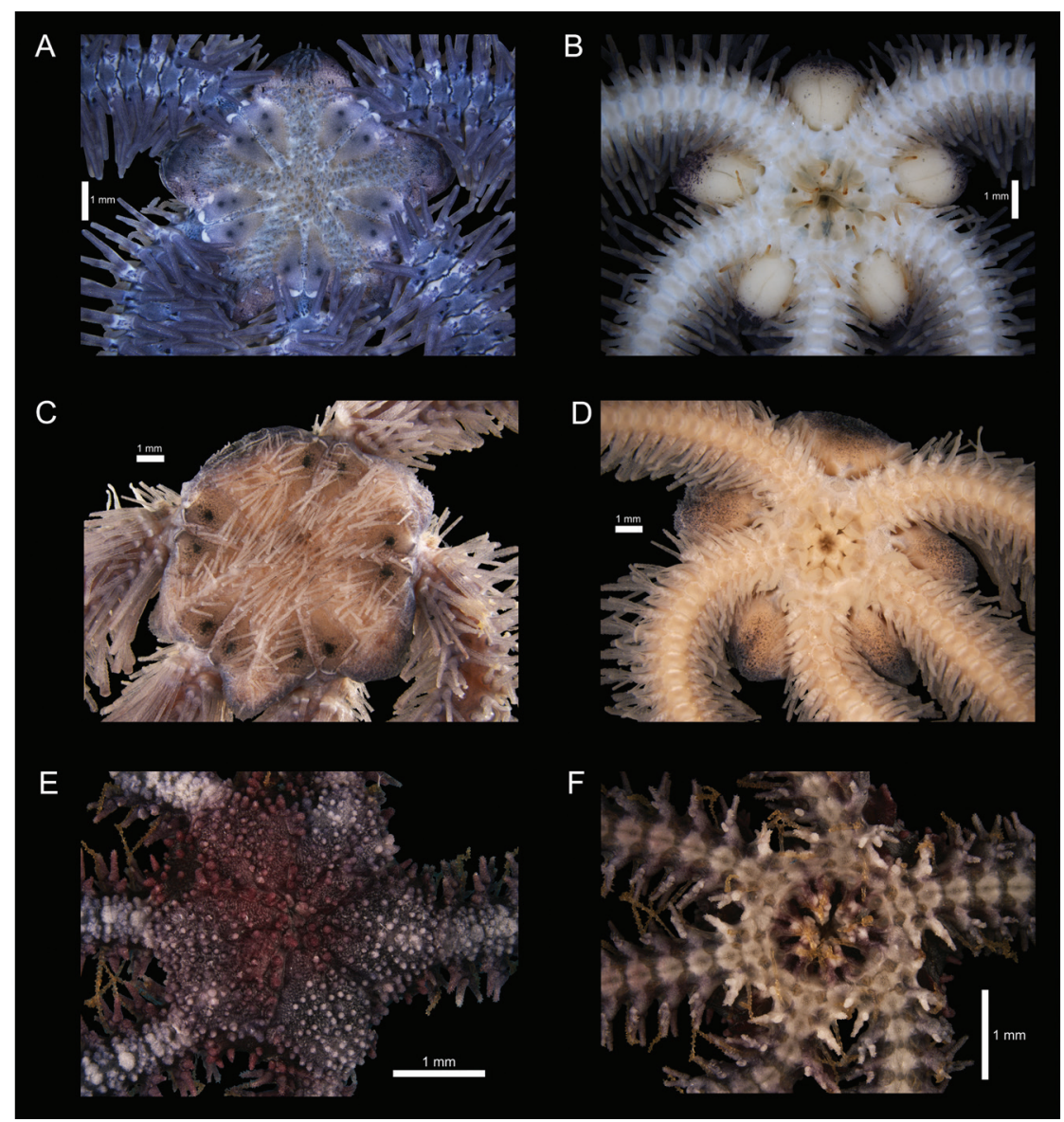

Fig. 6. Ophiothrix sp. (ICML-UNAM 18360). A. Vista dorsal. B. Vista ventral. Ophiothrix (Ophiothrix) spiculata (ICML-UNAM 11947). C. Vista dorsal. D. Vista ventral. Ophiothela mirabilis (ICML-UNAM 12155). E. Vista dorsal. F. Vista ventral.

Fig. 6. Ophiothrix sp. (ICML-UNAM 18360). A. Dorsal view. B. Ventral view. Ophiothrix (Ophiothrix) spiculata (ICML-UNAM 11947). C. Dorsal view. D. Ventral view. Ophiothela mirabilis (ICML-UNAM 12155). E. Dorsal view. F. Ventral view.

vivo: rosa-púrpura oscuro en la superficie dorsal y rosa pálido en la superficie ventral.

Distribución local: isla Cocinas, isla Pajarera, isla El Mamut, isla La Colorada, isla San Pedro.

Género Ophiothela Verrill, 1867

Ophiothela mirabilis Verrill, 1867

(Fig. 6E, 6F)

Descripción (ICML-UNAM 12155): DD $=3.9 \mathrm{~mm}, \mathrm{LB}=23.5 \mathrm{~mm}$. Disco rosetado cubierto por gránulos robustos de diferentes tamaños. Escudos radiales conspicuos, pero cubiertos por granulación. Interradio cubierto por piel. Mandíbula cubierta por piel. Escudos orales y adorales cubiertos por piel, formando un anillo alrededor de la boca. Aproximadamente 10 papilas dentales redondeadas en forma de racimo. Seis brazos (raramente cinco) enrollados. Placas dorsales de los brazos cubiertas por gránulos de diferentes tamaños. Placas ventrales de los brazos cubiertas por piel, más anchas que largas. De cinco a seis espinas aserradas, culminando en la parte distal en forma de gancho; la espina más dorsal es la más pequeña. Sin escamas tentaculares. Coloración in vivo: púrpura o rojiza-rosácea. 
Distribución local: isla Cocinas, isla Pajarera, isla La Colorada, isla La Negrita.

\section{DISCUSIÓN}

En este trabajo se reportan 19 especies de ofiuroideos distribuidas en dos órdenes, siete familias y 11 géneros para la bahía de Chamela, Jalisco, México. La familia con mayor representación es Ophiodermatidae, con dos géneros y cinco especies; actualmente, los estudios más recientes de esta familia son descripciones de nuevas especies y delimitación de especies (Weber, Stöhr, \& Chenuil, 2018; Stöhr, Weber, Boissin \& Chenuil, 2020). Por otro lado, la familia Ophionereididae fue la menos diversa al presentar solo una especie de un solo género. La especie con mayor abundancia fue Ophiactis savignyi con 833 especímenes y la de menor abundancia fue Ophioderma sp. con solo un individuo. Del total de especies se incluyen siete nuevos registros para el área de estudio (Diopederma daniana, Ophiocnida hispida, Ophiolepis pacifica, Ophiolepis plateia, Ophioderma teres var. unicolor, Ophiophragmus sp. y Ophiothrix sp.) los cuales amplían el conocimiento sobre la distribución local y regional.

Diopederma daniana fue recolectada en bahía de Chamela en el año 1939 a una profundidad de $24 \mathrm{~m}$, y se encuentra depositada en el USNM (USNM E 10033). A pesar de estar depositada en esta colección desde el siglo pasado, su registro nunca había sido publicado por lo que su adición en este trabajo representa un registro nuevo importante para el área de estudio. El método de recolecta para esta especie fue usando probablemente una draga o una red camaronera a bordo del buque oceanográfico Stranger M/S a 18-24 m de profundidad. Debido a los métodos de recolecta utilizados en el presente estudio (buceo SCUBA de 2 a $10 \mathrm{~m}$ ), el ambiente correspondiente a los fondos arenosos dentro de la bahía fue poco explorado, razón por la cual quizá esta especie no había sido encontrada durante los muestreos con anterioridad. Este sustrato posee un alto potencial de incidencia de ofiuroideos, por lo que se recomienda realizar exploraciones futuras intensivas en el mismo.

La primera recolecta de $O$. hispida para el estado de Jalisco la realizaron Granja-Fernández et al. (2014) en Cuastecomatito, una localidad ubicada a $54 \mathrm{~km}$ al sur de bahía de Chamela. Dentro del material recolectado en el presente trabajo, en el año 2018, se obtuvieron cinco especímenes previamente identificados como Ophiocnida cf. hispida debido a su similitud morfológica (ICML-UNAM 18343, ICML-UNAM 18344). Sin embargo, la revisión morfológica detallada de este material permitió corroborar su identidad taxonómica como $O$. hispida, siendo así un nuevo registro para la bahía de Chamela.

Ophiolepis plateia se distribuye en México, Costa Rica y Colombia (Ziesenhenne, 1940; Neira-Ortíz \& Cantera, 2005) particularmente en México, solo ha sido registrada en Jalisco (Granja-Fernández et al., 2015). Uno de los primeros registros de ofiuroideos para la costa de Jalisco fue el de esta especie, la cual fue descubierta por primera vez en la bahía de Tenacatita, Jalisco en los años 1935 y 1939 (Ziesenhenne, 1940). Cabe destacar que, desde su descripción, O. plateia no había sido recolectada sino hasta el año 2013 (en la bahía de Chamela), fecha que también representa su última recolecta. Esta especie, al igual que $D$. daniana, habita en sedimentos finos, lo cual nuevamente, denota la importancia de una mayor prospección en este tipo de sustrato. Por otro lado, el primer registro de O. pacifica para el estado de Jalisco lo realizó Caso (1951), recolectando únicamente un ejemplar en Puerto Vallarta, Jalisco. El descubrimiento de $O$. pacifica para la bahía de Chamela data de una recolecta de 2013, pero no había sido reportado en ninguna publicación para el área de estudio, siendo así un nuevo registro para la bahía. Con respecto a $O$. variegata se tienen dos registros en la literatura: el primero fue por parte de Clark (1940) y el segundo fue por Luke (1982), ambas campañas se realizaron con buques oceanográficos. A pesar de estos registros, O. variegata no se recolectó durante el presente trabajo dentro de la bahía, debido 
probablemente a que las especies de la familia Ophiolepididae tienen una afinidad a zonas arenosas (Hendler \& Turner, 1987).

Los registros de $O$. teres var. unicolor habían sido mal identificados y confundidos con Ophioderma teres. Granja-Fernández (2019) describe las diferencias más importantes entre estos dos taxa: $O$. teres presenta escudos radiales cubiertos o semi descubiertos, escudos orales más largos que anchos, placas ventrales de los brazos ligeramente más largas que anchas y disco moteado. Mientras que, $O$. teres var. unicolor presenta escudos radiales descubiertos, escudos orales ligeramente más anchos, placas ventrales mucho más largas que anchas y disco con reticulaciones negras.

Adicionalmente, se reportaron dos especies (Ophiophragmus sp. y Ophiothrix sp.) que podrían representar nuevas especies para la ciencia. Las identificaciones previas de estas especies eran erróneas debido a que comparten características con las especies congéneres respectivamente (Ophiophragmus lonchophorus, O. paucispinus y Ophiothrix (Ophiothrix) rudis) por lo que su correcta corroboración y descripción incrementará el total de especies reportadas, no solo para la bahía de Chamela, sino para el estado de Jalisco y el Pacífico Oriental Tropical. Por otro lado, el género Ophiophragmus presenta dos especies para el estado de Jalisco: el primer registro lo realizó Ziesenhenne (1940), al describir a O. lonchophorus, en la bahía de Tenacatita, Jalisco; el segundo registro pertenece a $O$. marginatus, realizado por Ríos-Jara et al. (2013). Durante las campañas de muestreo de este trabajo en el año 2018 se recolectaron dos especímenes de Ophiophragmus sp., los cuales difieren en los caracteres morfológicos externos de las dos especies antes mencionadas. Se propone realizar un mayor esfuerzo de muestreo usando técnicas de extracción en sedimentos arenosos para poder recolectar un mayor número de organismos y así poder corroborar su identidad taxonómica o bien, la probable descripción de una nueva especie. Finalmente, para Ophiothrix sp. actualmente se está realizando una revisión morfológica integrativa de la especie para corroborar su identidad taxonómica. Anteriormente, los registros con esta morfología habían sido identificados como O. (Ophiothrix) rudis; esto podría atribuirse a la información existente para discernir entre las especies del género reportadas para aguas someras del Pacífico mexicano. Actualmente, uno de los trabajos más utilizados para la identificación de especies es la clave dicotómica presente en el trabajo de Laguarda-Figueras, Escandón-Flores, Solís-Marín, HernándezHerrejón, y Durán-González (2011) donde Ophiothrix (Ophiothrix) spiculata y $O .(O$. rudis se diferencian entre sí por la ornamentación del disco y de las espinas. A pesar de esto, las distinciones entre géneros y especies dentro de la familia han sido problemática dada la gran variabilidad morfológica de muchas de las especies (Clark, 1966; Hendler, 2006).

Dentro del presente listado se comparten nueve especies con el trabajo de Ríos-Jara et al. (2013) (O. savignyi, O. simplex, O. aethiops, $O$. alexandri, O. variegatum, O. panamense, O. annulata, O. mirabilis y O. (Ophiothrix) spiculata), el cual era el más completo hasta el momento. Por otro lado, el amfiúrido $O$. marginatus fue la única especie reportada por Ríos-Jara et al. (2013) la cual no fue encontrada durante el trabajo de campo o las visitas a las colecciones científicas. La única especie con morfología similar a la especie anterior fue Ophiophragmus sp., la cual posiblemente corresponda a una nueva especie. Las diferencias encontradas entre ambos trabajos se pueden atribuir a que a partir del año 2016 se incrementó el esfuerzo en la captura de especies de ofiuroideos en la zona, y a que durante el presente estudio se incrementó la diversidad de hábitats y sustratos en los cuales se muestrearon ofiuroideos (e.g. madera sumergida, arena, debajo de rocas y algas vs zona intermareal, submareal y rocosa).

La morfología de las mandíbulas de los ofiuroideos encontrados en este trabajo está basada en la nueva propuesta de Hendler (2018). De acuerdo con la anterior, la mandíbula de algunas especies del género Ophiactis pueden poseer una escama bucal, una espina 
del escudo adoral y en algunos casos también, una segunda espina del escudo adoral. Pese a lo anterior, la denominación de esas estructuras no es explícita en Hendler (2018) por lo que no fue posible asignarlas a $O$. savignyi ni a $O$. simplex durante este trabajo. Se sugiere hacer un estudio o una reevaluación de forma más detallada de la mandíbula de estas especies (familia Ophiactidae) con la finalidad de establecer las estructuras mandibulares correspondientes.

En la bahía de Chamela tanto los estudios de ofiuroideos (Clark, 1940; Caso, 1951; Luke, 1982; López-Uriarte et al., 2009; Ríos-Jara et al., 2013; Granja-Fernández et al., 2014; Granja-Fernández et al., 2017) como los registros de otros equinodermos han sido escasos (Ríos-Jara et al., 2013). Con los nuevos registros encontrados durante el trabajo de campo y revisión de muestras de colecciones científicas, se aumenta el listado de equinodermos para la bahía de Chamela a 48 especies. Cabe destacar que, el presente trabajo es el primero en presentar un listado taxonómico completo para la clase Ophiuroidea en la bahía de Chamela, Jalisco, así como la primera clave taxonómica para su identificación. Esta clave puede ser empleada en más localidades dentro de la franja litoral del Pacífico tropical mexicano debido a que las especies presentes en este trabajo tienen una amplia distribución a lo largo de esta región (Granja-Fernández, 2019).

De las 125 especies de ofiuroideos reportadas para el Pacífico mexicano (Granja-Fernández et al., 2015), 19 especies se encuentran en la bahía de Chamela y equivalen al $15.2 \%$ de la diversidad total reportada. En el caso particular del estado de Jalisco, en el más reciente recuento de ofiuroideos para el estado, GranjaFernández et al. (2017) reportaron 26 especies. Por lo que se concluye que las islas de la bahía de Chamela albergan el $73 \%$ de su biodiversidad. Las numerosas islas de bahía de Chamela, así como su amplia heterogeneidad ambiental (e.g. rocas, sedimento, algas) podrían ser la razón por la cual el área posee una importante biodiversidad de ofiuroideos. Los trabajos de taxonomía que actualmente se realizan con equinodermos son la base para muchos trabajos de biogeografía y ecología. Asimismo, son de suma importancia para elaborar o complementar programas de manejo de áreas naturales protegidas, donde la parte marina casi siempre tiene un vacío de información respecto a invertebrados marinos.

\section{AGRADECIMIENTOS}

Los autores agradecen a Alicia Durán (Laboratorio de Sistemática y Ecología de Equinodermos, ICML, UNAM) por el acceso al material depositado en la Colección Nacional de Equinodermos, ICML, UNAM. A Penny Benson y Adam Baldinger (MCZ), y a David Pawson, Chad Walter, Geoff Keel, Jon Nurenburg y Paul Greenhall (USNM), Gordon Hendler y Cathy Groves (LACM) y Tom Schiotte y Jorgen Oelsen (NHMD) por el apoyo en las visitas a las colecciones. A Susana Guzmán Gómez (Laboratorio de Microscopía y Fotografía de la Biodiversidad II, Instituto de Biología, UNAM) por la toma de fotografías multifocales en el microscopio estereoscópico Leica Z16 APOA. A Renata Alitto y Tania Pineda por la discusión sobre algunas estructuras morfológicas. CACV (becario 666781) agradece al Consejo Nacional de Ciencia y Tecnología (CONACyT) por la beca de doctorado 722925. GIRC (becario 492379) agradece al Consejo Nacional de Ciencia y Tecnología (CONACyT) por la beca de doctorado 281719.

\section{RESUMEN}

\section{Los ofiuroideos (Echinodermata: Ophiuroidea) de la bahía de Chamela, Jalisco, México}

Introducción: La bahía de Chamela está ubicada en la parte central de la costa del estado de Jalisco, México y es un importante área natural protegida. Pese a ello, el estudio de los equinodermos en la zona es incipiente. Objetivo: Proporcionar una lista de verificación actualizada, descripciones de las especies y una clave taxonómica de los ofiuroideos de la bahía de Chamela. Métodos: Recolectamos en la bahía de 1998 a 2018, y también analizamos información de la literatura y especímenes en colecciones científicas internacionales. Resultados: se reportan 19 especies de ofiuroides para el área de estudio, distribuidas 
en dos órdenes, siete familias y 11 géneros. Ocho de estos corresponden a nuevos registros para el área y tres posiblemente representan nuevas especies. La biodiversidad de ofiuroideos en la bahía de Chamela representa el 15.2\% de las especies de ofiuroides del Pacífico mexicano y el 73\% de Jalisco. El número de equinodermos conocidos en la bahía de Chamela aumenta a 48 especies. Conclusiones: Se presenta el listado más completo de la clase Ophiuroidea en la bahía de Chamela, Jalisco. La información proporcionada se puede utilizar para otras áreas del Pacífico oriental. La bahía de Chamela es importante en términos de riqueza de especies debido a su heterogeneidad ambiental y numerosas islas.

Palabras clave: clave taxonómica; Pacífico mexicano; biodiversidad; nuevos registros; morfología; taxonomía.

\section{REFERENCIAS}

Caso, M.E. (1951). Contribución al conocimiento de los ofiuroideos de México. 1. Algunas especies de ofiuroideos litorales. Anales Instituto de Biología, Universidad Nacional Autónoma de México, 22(1), 219-312.

Clark, A.M. (1966) Notes on the family Ophiotrichidae (Ophiuroidea). Journal of Natural History Series, 13(9), 106-108.

Clark, H.L. (1913). Echinoderms from Lower California, with descriptions of new species. Bulletin of the American Museum of Natural History, 32, 185-236.

Clark, H.L. (1940). Notes on Echinoderms from the West Coast of Central America. Eastern Pacific Expeditions of the New York Zoological Society. Zoologica, 25, 331-352.

Fell, H.B. (1960). Synoptic keys to the genera of Ophiuroidea. Zoology Publications from Victoria University of Wellington, 20, 1-44.

Frontana-Uribe, S. \& Solís-Marín, F.A. (1999). Listado sistemático de los equinodermos (Echinodermata) de la Isla Cocina, Jalisco, México. Tepic, México: Resúmenes del XV Congreso Nacional de Zoología y VII Reunión Nacional de Malacología y Conquiliología.

Granja-Fernández, M.R. (2019). Ofiuroideos (Echinodermata: Ophiuroidea) del Pacífico Oriental Tropical (Tesis doctoral). Universidad Autónoma Metropolitana, México.

Granja-Fernández, R., Herrero-Pérezrul, M.D., LópezPérez, R.A., Hérnandez, L., Rodríguez-Zaragoza, F.A., Jones, R.W., \& Pineda-López, R. (2014). Ophiuroidea (Echinodermata) from coral reefs in the Mexican Pacific. ZooKeys, 406, 101-145.

Granja-Fernández, R., Herrero-Pérezrul, M.D., LópezPérez, R.A., Hernández-Morales, A., \& Rangel-Solís,
P.D. (2015). A literature review of the Ophiuroidea (Echinodermata) from the Pacific coast of Mexico. Revista de Biología Tropical, 63(2), 37-47.

Granja-Fernández, R., Rodríguez-Troncoso, A.P., HerreroPérezrul, M.D., Sotelo-Casas, R.C., Flores-Ortega, J.R., Godínez-Domínguez, E., \& Cupul-Magaña, A.L. (2017). Ophiuroidea (Echinodermata) from the Central Mexican Pacific: an updated checklist including new distribution records. Marine Biodiversity, 47(1), 167-177.

Gray, J.E. (1840). A synopsis of the genera and species of the Class Hypostoma (Asterias, Linnaeus). Annals and Magazine of Natural History, Zoology, Botany, and Geology, 6, 175-184.

Hendler, G. \& Turner, R.L. (1987). Two new species of Ophiolepis (Echinodermata: Ophiuroidea) from the Caribbean Sea and Gulf of Mexico: With notes on ecology, reproduction and morphology. Contributions in Science, 395, 1-14.

Hendler, G. (2006). Two new brittle star species of the genus Ophiothrix (Echinodermata: Ophiuroidea: Ophiotrichidae) from coral reefs in the southern Caribbean Sea, with notes on their biology. Caribbean Journal of Science, 41, 583-599.

Hendler, G. (2018). Armed to the teeth: a new paradigm for the buccal skeleton of brittle stars (Echinodermata: Ophiuroidea). Contributions in Science, 526, 189-311.

Laguarda-Figueras, A., Escandón-Flores, N., Solís-Marín, F.A., Hernández-Herrejón, L.A. \& Durán-González, A. (2011). Los ofiuroideos del Golfo de California. Ciudad de México, México: SEMARNAT, INECC.

Le Conte, J.L. (1851). Zoological Notes. Proceedings of the Academy of Natural Science of Philadelphia, 5, 316-319.

Ljungman, A.V. (1866). Om några nya arter af Ophiurider. Öfversigt af Kongliga Vetenskaps Akademiens Förhandlingar, 23(6), 163-166.

Ljungman, A.V. (1867). Ophiuroidea viventia huc usque cognita eneumerat. Öfversigt af Kongl. VetenskapsAkademiens Förhandlingar, 23, 303-336.

López-Uriarte, E., Ríos-Jara, E., Galván-Villa, C., JuárezCarrillo, E., Enciso-Padilla, I., Robles-Jarero, E. \& Pérez-Peña, M. (2009). Macroinvertebrados bénticos del litoral somero Punta La Rosada, Bahía Chamela, Jalisco. Scientia-CUCBA, 11(1-2), 57-68.

Luke, S.R. (1982). Catalog of the benthic invertebrate collections of the SCRIPPS Institution of Oceanography, Echinodermata. California, USA: SCRIPPS Institution of Oceanography Reference Series, no. 82-5, University of California. 
Lütken, C.F. (1856). Bidrag til Kundskabom Slangestjerne. III. Bidrag til Kunskab om Ophiurerne ved CentralAmerikas Vestkyst. Videnskabelige Meddelelser fra Dansk Naturhistorisk Forening 1856, 1-26.

Lütken, C.F. (1859). Addimenta af historiam Ophiuridarum. Kongelige Danske Videnskabernes Selskab Biologiske Skrifter, 5(5), 177-271.

Lyman, T. (1865). Ophiuridae and Astrophytidae. Illustrated Catalogue of the Museum of Comparative Zoology, at Harvard College, 1, 1-200.

Matsumoto, H. (1915). A new classification of the Ophiuroidea: with description of new genera and species. Proceedings of the Academy of Natural Sciences of Philadelphia, 68, 43-92.

Miranda, A., Ambriz, G., Valencia, D., Sánchez, M. \& Szekely, A. (2011). Programa de Manejo del Santuario de las Islas de Chamela: Islas La Pajarera, Cocinas, Mamut, Colorada, San Pedro, San Agustín, San Andrés y Negrita, e islotes Los Anegados, Novillas, Mosca y Submarino. Jalisco. México. Ciudad de México, México: Comisión Nacional de Áreas Naturales Protegidas.

Müller, J.H. \& Troschel, F.H. (1840). Fortgesetzte Bemerkungen über die Gattungen der Asteriden. En A.F.A. Weigmann (Ed.), Archiv für Naturgeschichte (pp. 367-368). Berlin, Alemania: Nicolai.

Müller, J.H. \& Troschel, F.H. (1842). System der Asteriden. Berlin, Alemania: F. Vieweg \& Sohn, Braunschweig.

Neira-Ortíz, R. \& Cantera, J.R. (2005) Composición taxonómica y distribución de las asociaciones de equinodermos en los ecosistemas litorales del Pacífico Colombiano. Revista de Biología Tropical, 53(3), 195-206.

O’Hara, T.D., Hugall, A.F., Thuy, B., Stöhr, S. \& Martynov, A. (2017) Restructuring higher taxonomy using broad-scale phylogenomics: The living Ophiuroidea. Molecular Phylogenetics and Evolution, 107, 415-430.

O’Hara, T.D., Stöhr, S., Hugall, A.F., Thuy, B. \& Martynov, A. (2018). Morphological diagnoses of higher taxa in Ophiuroidea (Echinodermata) in support of a new classification. European Journal of Taxonomy, $416,1-35$.

Ríos-Jara, E., Galván-Villa, C., Rodríguez-Zaragoza, F., López-Uriarte, E., Bastida-Izaguirre, D., \& SolísMarín, F.A. (2013). Los Equinodermos (Echinodermata) de Bahía de Chamela, Jalisco, México. Revista Mexicana de Biodiversidad, 84(1), 263-279.

Stöhr, S., O’Hara, T., Thuy, B. (2020). World Ophiuroidea Database. Retrieved from http://www.marinespecies. org/ophiuroidea

Stöhr, S., Weber, A.A.T., Boissin, E., \& Chenuil, A. (2020). Resolving the Ophioderma longicauda (Echinodermata: Ophiuroidea) cryptic species complex: five sisters, three of them new. European Journal of Taxonomy, 600, 1-37.

Verrill, A.E. (1867). Notes on the Radiata in the Museum of Yale College, with descriptions of new genera and species. No. 2. Notes on the Echinoderms of Panama and West Coast of America, with descriptions of new genera and species. Transactions of the Connecticut Academy of Arts and Sciences, 1, 251-322.

Weber, A., Stöhr, S., \& Chenuil, A. (2018) Species delimitation in the presence of strong incomplete lineage sorting and hybridization: lessons from Ophioderma (Ophiuroidea: Echinodermata). Molecular Phylogenetics and Evolution, 131, 138-148.

Ziesenhenne, F.C. (1940). New Ophiurans of the Allan Hancock Pacific Expeditions. Allan Hancock Pacific Expeditions, 8(2), 9-52. 